\title{
Spectral superbroadening of self-focused picosecond laser pulses in $D_{\mathbf{2}} \mathrm{O}$
}

\author{
A. PENZKOFER, A. BEIDOUN, H. - J. LEHMEIER* \\ Naturwissenschaftliche Fakultät II-Physik, \\ Universität Regensburg, W-8400 Regensburg, Germany
}

Received 12 November; accepted 16 December 1992

Intense picosecond light pulses of a mode-locked Nd : glass laser at $\lambda_{L}=1054 \mathrm{~nm}$ (fundamental wavelength) and $\lambda_{S H}=527 \mathrm{~nm}$ (second harmonic wavelength) are passed through a sample of $D_{2} O$ under self-focusing conditions. Spectrally structured superbroadened, spatially bell-shaped emission in the forward direction is obtained. Primary generation processes are pump-pulse-degenerate stimulated parametric four-photon interaction $\left(\omega_{1}+\omega_{1} \rightarrow \omega_{3}+\omega_{4}\right)$ and stimulated Raman scattering $\left(\omega_{1} \rightarrow \omega_{R}+\omega_{l}\right)$, which occur concurrently $\left(\omega_{1}=\omega_{L}\right.$ or $\omega_{S H}$ angular pump frequency, $\omega_{R}=$ first Stokes frequency, $\omega_{v}=$ vibrational frequency, $\omega_{3}=$ signal frequency, $\omega_{4}=$ idler frequency). The parametric four-photon interaction occurs under collinear non-phase-matched conditions and under longitudinally phase-matched, transversally non-phase-matched (Čerenkov-like) conditions. Subsequent interaction processes are pump-pulse-nondegenerate four-photon interaction of the type $\omega_{1}+\omega_{R} \rightarrow \omega_{3^{\prime}}+\omega_{4^{\prime}}$, coherent antiStokes Raman scattering (CARS, $\omega_{1}+\omega_{1}-$ $\left.\omega_{4} \rightarrow \omega_{3}\right)$, inverse Raman scattering $\left(\omega_{A} \rightarrow \omega_{1}+\omega_{v}\right)$, and cascading light up-conversion of the type $\omega_{1}+\omega_{(i)}-\omega_{R} \rightarrow \omega_{(i+1)}$.

\section{Introduction}

Light continua of duration and polarization similar to those of the pump sources are generated when intense nanosecond [1, 2], subnanosecond [3], picosecond [4-41], or femtosecond [42-46] light pulses pass through dielectric media in gaseous [33, 44, 47], liquid [1-3, $8-28,30,35,38-43,45,46]$, or solid state $[4-11,15-17,19,23,26,29,31,32,34,36,37]$ form. The generation of light continua is reviewed in [48-52]. Weakly dispersive media with high stimulated Raman scattering threshold (liquids and glasses with broadband Raman transitions, crystals with forbidden Raman transitions, rare gases) are favourable for the generation of smooth broadband spectra [21, 23, 24, 35, 48]. Contributions from stimulated Raman scattering are generally avoided in femtosecond pulse continuum generation [42-47] because the Raman scattering process becomes transient [53, 54]. Theoretical treatments of light continuum generation are given in [22, 23, 41, 55-63]. Several mechanisms of spectral superbroadening have been discussed as self-phase modulation $[4-6,10,12-14,16,19,36,42,49]$, dielectric breakdown-enhanced self-phase modulation $[22,27,55]$, combined self-phase modulation and stimulated Raman interaction [20-22, 24,

*Permanent address: DASA AG, W-8012 Ottobrunn, Germany. 
$25,35,36]$, combined cross-phase and self-phase modulation [34, 36-38], self-phase modulation of pulse amplitudes and phases together with self-steepening [59, 61], parametric four-photon interaction $[11,15-18,23,24,27,29,56,57]$, combination of parametric four-photon interaction with self-phase modulation $[23,36]$ and Raman scattering [30, $36,58,62,63]$, and Čerenkov radiation under self-focusing conditions [41]. Light continuum generation is most easily achieved under self-focusing and self-trapping conditions [64] where the beam focusing provides the necessary high laser intensities.

In this paper the generation of light continua in $\mathrm{D}_{2} \mathrm{O}$ is studied under self-focusing conditions. Picosecond pump pulses at $1054 \mathrm{~nm}$ (mode-locked $\mathrm{Nd}$ : glass laser, pulse duration $\Delta t_{L} \approx 6 \mathrm{ps}$ ) and at $\lambda_{L}=527 \mathrm{~nm}$ (second harmonic of mode-locked $\mathrm{Nd}$ : glass laser, $\Delta t_{S H} \approx 5 \mathrm{ps}$ ) are applied. Efficient stimulated Raman scattering occurs concurrently with the continuum generation. A characteristic spectral structuring of the superbroadened spectra is observed that is interpreted by an interplay of collinear nonphase-matched parametric four-photon interaction, longitudinally phase-matched, transversally non-phase-matched (Čerenkov-like) parametric four-photon interaction, and Stokes-antiStokes Raman coupling. Pump-pulse-degenerate four-photon interaction $\omega_{1}+\omega_{1} \rightarrow \omega_{3}+\omega_{4}\left(\omega_{1}=\omega_{L}\right.$ or $\omega_{S H}$ is pump pulse frequency), pump-pulse-nondegenerate four-photon interaction $\omega_{1}+\omega_{R} \rightarrow \omega_{3^{\prime}}+\omega_{4^{\prime}}$ ( $\omega_{R}$ is first Stokes Raman frequency), coherent antiStokes Raman scattering $\left(\omega_{1}+\omega_{1}-\omega_{4} \rightarrow \omega_{3}\right)$, inverse Raman scattering $\left(\omega_{A} \rightarrow \omega_{1}+\omega_{v}\right)$, and cascading longitudinally phase-matched light up-conversion $\omega_{1}+\omega_{(i)}-\omega_{R} \rightarrow \omega_{(i+1)}$ are involved. The four-photon interaction in self-focused filaments reduces the phase-matching requirement to longitudinal phase-matching and results in spatially bell-shaped light continuum emission in the forward direction.

\section{Experimental}

A schematic of the experimental setup is shown in Fig. 1. Picosecond light pulses are generated in an active (acoustooptic modulator IntraAction model ML-50Q) and passive (saturable absorber Kodak dye No. 9860) mode-locked Nd : phosphate glass laser [65]. The laser wavelength is $\lambda_{L}=1054 \mathrm{~nm}$ and the pulse duration is $\Delta t_{L} \approx 6 \mathrm{ps}$. Single pulses are selected in the rising part of the mode-locked pulse trains by a spark-gap-triggered Kerr shutter. The separated pulses are increased in energy in an $\mathrm{Nd}$ : phosphate glass amplifier. In the case of second-harmonic pulse excitation, the fundamental pulses are frequency-doubled in a CDA crystal (caesium dihydrogen arsenate $\mathrm{CsH}_{2} \mathrm{AsO}_{4}$, length $1 \mathrm{~cm}$, noncritical $90^{\circ}$ phase-matching at $34^{\circ} \mathrm{C}$ ) [66]. The second-harmonic pulse duration is $\Delta t_{S H} \approx 5 \mathrm{ps}$. Either the fundamental or second-harmonic pulses are focused to the $\mathrm{D}_{2} \mathrm{O}$ sample $\mathrm{S}$ (focal length of lens $\mathrm{L} 1$ is $25 \mathrm{~cm}$ unless stated otherwise). The sample length is $l=5 \mathrm{~cm}$. The input pulse energy is detected by photodetector PD1. Photodetector PD3 behind a laser or second-harmonic interference filter IF detects the spectrally unshifted transmitted pump pulse energy. The polarization of the generated Raman light and of the light continuum was determined by placing a polarizer between L2 and CL2 (not shown in Fig. 1). The polarization of the generated light was found to be parallel to the polarization of the input pump light. Different spectral regions of the generated spectra are registered with spectrometers SP1 and SP2, which are equipped with a photodiode array system and a vidicon system, respectively. The spectral sensitivity of the spectrometer systems is corrected by calibrating with a tungsten lamp of known colour temperature (Osram type HLX 64625 halogen-projection bulb at $12 \mathrm{~V}$ voltage, colour temperature 


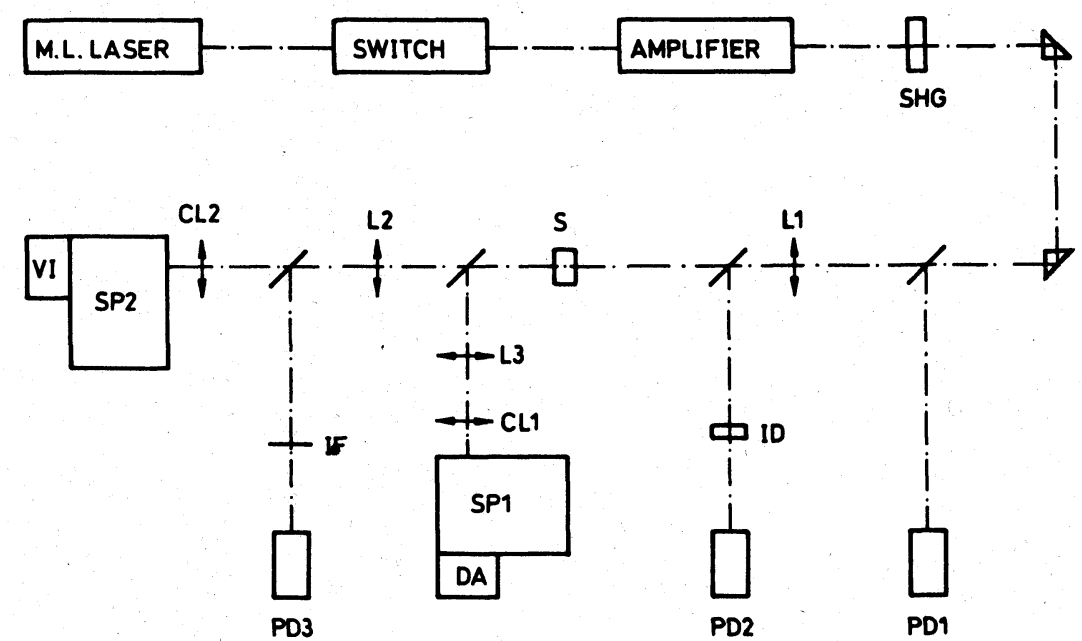

Figure 1 Experimental setup. SHG, CDA crystal for second-harmonic generation. L1 to L3, focusing lenses. S, $\mathrm{D}_{2} \mathrm{O}$ sample. $\mathrm{CL1}, \mathrm{CL2}$, cylindrical focusing lenses. PD1 to PD3, photodetectors. IF, laser interference filter (spectral transmission full-width at half-maximum is $\Delta \lambda_{I F}=7.6 \mathrm{~nm}$ and $5.5 \mathrm{~nm}$ for $\lambda_{L}=1054 \mathrm{~nm}$ and $\lambda_{S H}=527 \mathrm{~nm}$, respectively). SP1, SP2, $25-\mathrm{cm}$ grating spectrometers. DA, diode array system. VI, vidicon system. ID, two-photon absorbing rutile crystal for pump pulse peak intensity detection at $\lambda_{S H}=527 \mathrm{~nm}$ [72] (applied only in experiments where an $80-\mathrm{cm}$ input focusing lens L1 was used).

$3450 \mathrm{~K})$ [67]. Spectral energy densities $I(\lambda)$ are measured. In most of the following figures, $I(\tilde{\nu})=I(\lambda)|\mathrm{d} \lambda / \mathrm{d} \tilde{\nu}|=I(\lambda) / \tilde{\nu}^{2}=\lambda^{2} I(\lambda)$ is displayed $\left(\tilde{\nu}=\lambda^{-1}\right)$.

\section{Results}

\subsection{Fundamental laser excitation}

Three examples of pulse spectra obtained are displayed in Figs 2 to 4 . The input pulse energies were $W_{L, \text { in }} \approx 900 \mu \mathrm{J}$ (Fig. 2), $\approx 1.2 \mathrm{~mJ}$ (Fig. 3), and $90 \mu \mathrm{J}$ (Fig. 4). The ratio of the central spectral pulse energy $W\left(\Delta \lambda_{I F}\right)$ passing the interference filter IF to the input pulse energy $W_{L, \text { in }}$ was $T_{c}=W\left(\Delta \lambda_{I F}\right) / W_{L, \text { in }} \approx 0.23$ (Fig. 2), $\approx 0.11$ (Fig. 3) and $\approx 0.15$ (Fig. $\left.4, \Delta \lambda_{I F} \approx 7.6 \mathrm{~nm}\right)$. The spectral energy content in the short-wavelength range $\lambda<1000 \mathrm{~nm}\left(\tilde{\nu}-\tilde{\nu}_{L}>500 \mathrm{~cm}^{-1}\right)$ is roughly $W(\lambda<1000 \mathrm{~nm}) / W_{L, \text { in }} \approx 0.015$ in all three cases. The missing energy content is thought to be Stokes shifted and mainly converted to stimulated Stokes Raman light.

The structuring of the spectra of Figs 2 to 4 is thought to be caused by CARS-like first Stokes-first antiStokes coupling and cascading antiStokes up-conversion. $\tilde{\nu}_{A}=\tilde{\nu}_{L}+\tilde{\nu}_{v}$ marks the first antiStokes frequency position $\left(\tilde{\nu}_{v}=2 \nu_{2 s}^{\prime}=2380 \mathrm{~cm}^{-1}\right.$ is used [68], see below). The spectral shape around $\tilde{\nu}_{A}$ is typical of coherent antiStokes Raman scattering [69] and Raman resonant (difference frequency resonant) stimulated parametric fourphoton interaction. The spectral shift of the first antiStokes peak is less than $\tilde{\nu}_{v}$. The repeated short-wavelength structuring is thought to be due to repetitive antiStokes upconversion of the type $\tilde{\nu}_{l}+\tilde{\nu}_{(i)}-\tilde{\nu}_{R} \rightarrow \tilde{\nu}_{(i+1)}$ where $\tilde{\nu}_{R}=\tilde{\nu}_{l .}-\tilde{\nu}_{v}$ is the frequency of the tirst Stokes component, and $i_{(i)}$ represents a frequency component present after the $i$ th repetition step which is transferred to $\tilde{\nu}_{(i+1)}$ in the $(i+1)$ th step. The frequency positions $\tilde{\nu}_{i A}=\tilde{\nu}_{L}+\mathrm{i} \tilde{\nu}_{v}$ are indicated in Figs 2 to 4. 


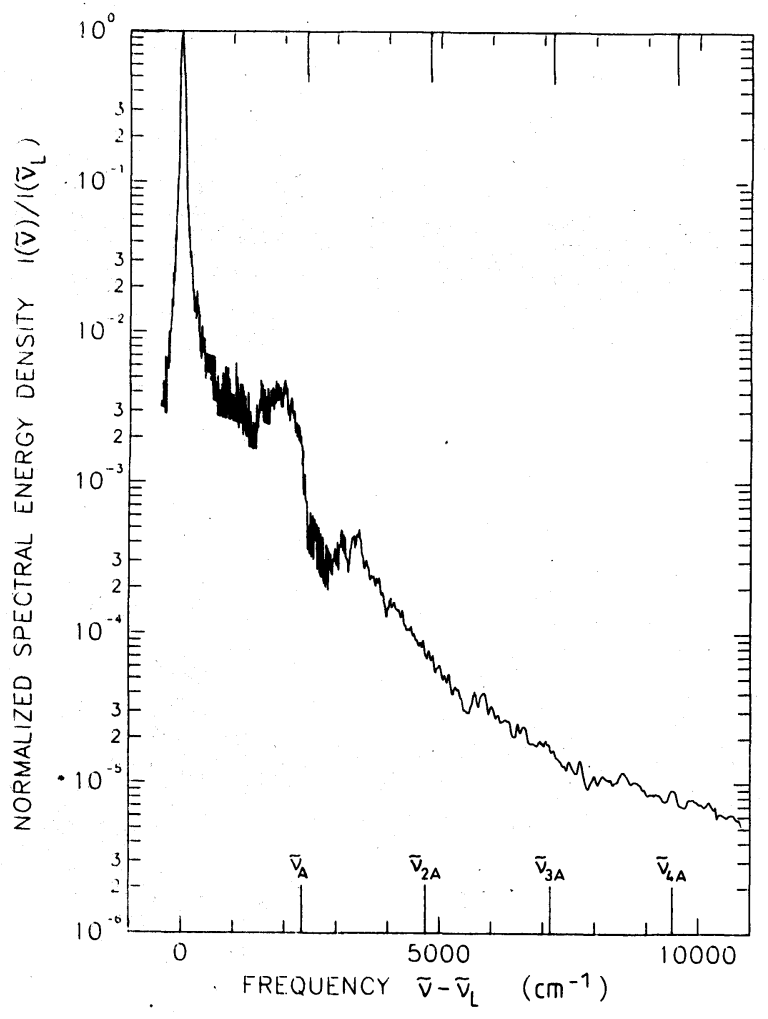

Figure 2 Output spectrum behind $\mathrm{D}_{2} \mathrm{O}$ sample. Fundamental laser excitation. Spectra of SP1 and SP2 are connected at $\tilde{\nu}-\tilde{\nu}_{L}=3500 \mathrm{~cm}^{-1}$. Input pulse energy $W_{L, \text { in }} \approx 900 \mu \mathrm{J}$. Central spectral pulse transmission $T_{c} \approx 0.23$.

In a recent pump and probe experiment on singlet excited state absorption of eosin Y [70] (pump pulses: second-harmonic light at $527 \mathrm{~nm}$; probe pulses: light continuum generated by 1054-nm laser excitation), one shot out of a series of about 300 shots delivered the spectrum displayed in Fig. 5. Above a smooth continuum a series of four roughly equally spaced peaks is present. The spectral separation of the peaks is $\delta \tilde{\nu}_{A} \approx 2070 \mathrm{~cm}^{-1}$. This separation is less than $\tilde{\nu}_{v}$. The first peak around $\tilde{\nu}-\tilde{\nu}_{L} \approx 2300 \mathrm{~cm}^{-1}$ is thought to be caused by CARS-like first antiStokes Raman scattering, while the other peaks are thought to be caused by cascading antiStokes up-conversion of the type $\tilde{\nu}_{L}+$ $\tilde{\nu}_{(i)}-\tilde{\nu}_{4^{\prime}} \rightarrow \tilde{\nu}_{(i+1)}$ where $\tilde{\nu}_{4^{\prime}}=\tilde{\nu}_{L}-\delta \tilde{\nu}_{A}$ is the frequency of an expected intense pulse generated by parametric four-photon interaction of the type $\tilde{\nu}_{L}+\tilde{\nu}_{R} \rightarrow \tilde{\nu}_{3^{\prime}}+\tilde{\nu}_{4^{\prime}}$.

The spectral emission occurs in the forward direction within a divergence angle of $\Delta \theta \approx 0.05 \mathrm{rad}$ (FWHM). The measured half-divergence angles are displayed as circles in Fig. 15. The cross-sectional radial distribution of the spectrally superbroadened pulses is bell-shaped. Conical rainbow-like emission was not observed. In Fig. 6 some crosssectional distributions at selected wavelengths are presented (spectra selected with interference filters, distributions registered with a photodiode array at a distance of $12 \mathrm{~cm}$ behind the sample). The radial width of the selected spectra is roughly independent of the frequency. Visual inspection of the emission on a white paper card indicated a smooth white cross-sectional light distribution with a bluish hue at the margin.

\subsection{Second-harmonic laser excitation}

Spectral distributions obtained by second-harmonic pumping are displayed in Figs 7 to 10 . 


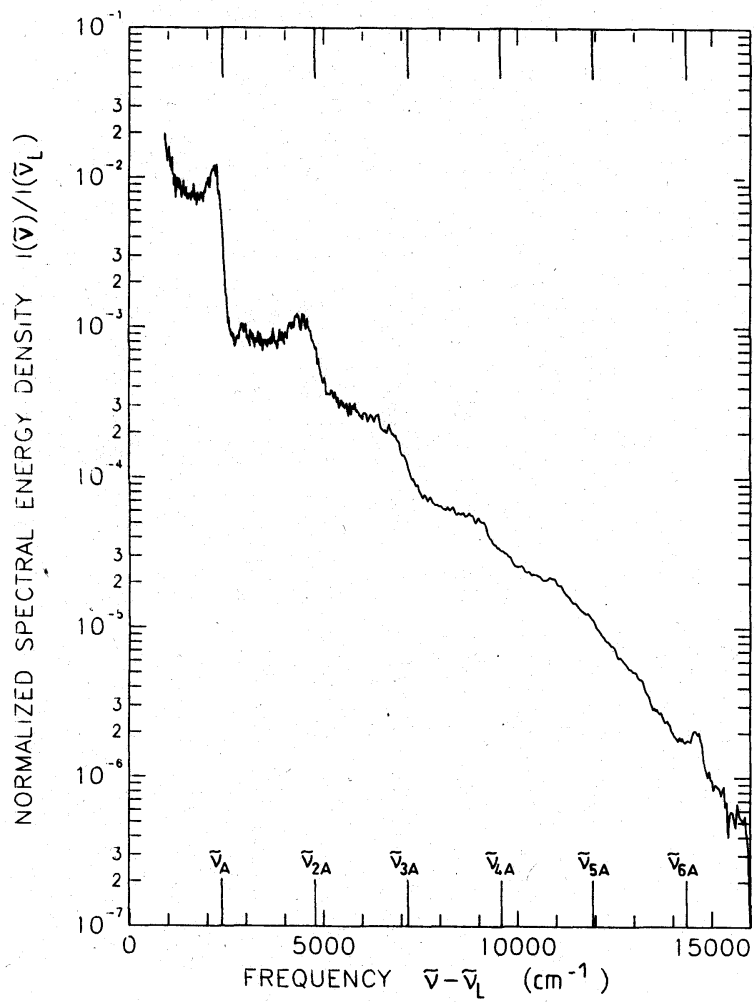

Figure 3 Output spectrum behind $\mathrm{D}_{2} \mathrm{O}$ sample. Fundamental laser excitation. Connection of spectra of SP1 and SP2 at $\tilde{\nu}-\tilde{\nu}_{L}=6000 \mathrm{~cm}^{-1} . W_{L, \text { in }} \approx 1.2 \mathrm{mj} . T_{c} \approx$ 0.11 .

In Fig. 7 the spectral shape of an input second-harmonic pump pulse (Fig. 7a, experimental setup of Fig. 1 without $\mathrm{D}_{2} \mathrm{O}$ ) and the spectral shape of a pulse behind the $\mathrm{D}_{2} \mathrm{O}$ sample (Fig. 7b) are shown. The displayed input pulse is self-phase modulated (temporalspectral bandwidth product is $\Delta \nu_{L} \Delta t_{L} \approx 4.7, \Delta t_{L} \approx 5 \mathrm{ps}$ ). The bandwidth product of the output pulse shown is $\Delta \nu_{L} \Delta t_{L} \approx 5.2$. The spectral centre of the output pulse is sharpened up and the spectral wings are broadened compared to the input spectrum. The characteristic self-phase modulation structure of the input pulse is lost. The transmitted pulse energy content within a bandwidth of $5.5 \mathrm{~nm}$ (FWHM) centred around the secondharmonic wavelength of $\lambda_{L}=527 \mathrm{~nm}$ is $T_{c} \approx 0.35$. The self-phase modulated pulse parts seem to be predominantly converted to Stokes Raman light and continuum light, while unbroadened spectral components in the temporal and spatial periphery seem to remain unchanged and form the centrally steepened spectrum.

In Figs 8 and 9 three output pulse spectra are shown. The solid curve in Fig. 8 was obtained for an input pulse energy of $W_{S H}$, in $\approx 750 \mu \mathrm{J}$, and the energy transmission of the central spectral part was $T_{c} \approx 0.24$. The Stokes Raman peak resolves the two most intense vibrational lines of spectral shift $\delta \tilde{\nu}_{R}=2 \nu_{2 s}^{\prime}=2380 \mathrm{cin}^{-1}$ (symmetric Fermi resonance mode) and $\nu_{s s}^{\prime}=2486 \mathrm{~cm}^{-1}$ (symmetric O-D stretching mode) [68,71]. At the antiStokes side, $\tilde{\nu}_{A}$ indicates the frequency position of $\tilde{\nu}_{L}+\delta \tilde{\nu}_{R}$. The CARS-like first antiStokes signal peaks at a frequency shift of $\approx 2200 \mathrm{~cm}^{-1}$. In the region between $\tilde{\nu}_{L}+\delta \tilde{\nu}_{R}$ and $\tilde{\nu}_{L}-\delta \tilde{\nu}_{R}$ the energy content of the spectral components at the Stokes side is somewhat higher than the energy content of the spectral components at the antiStokes side. At the Stokes side the 


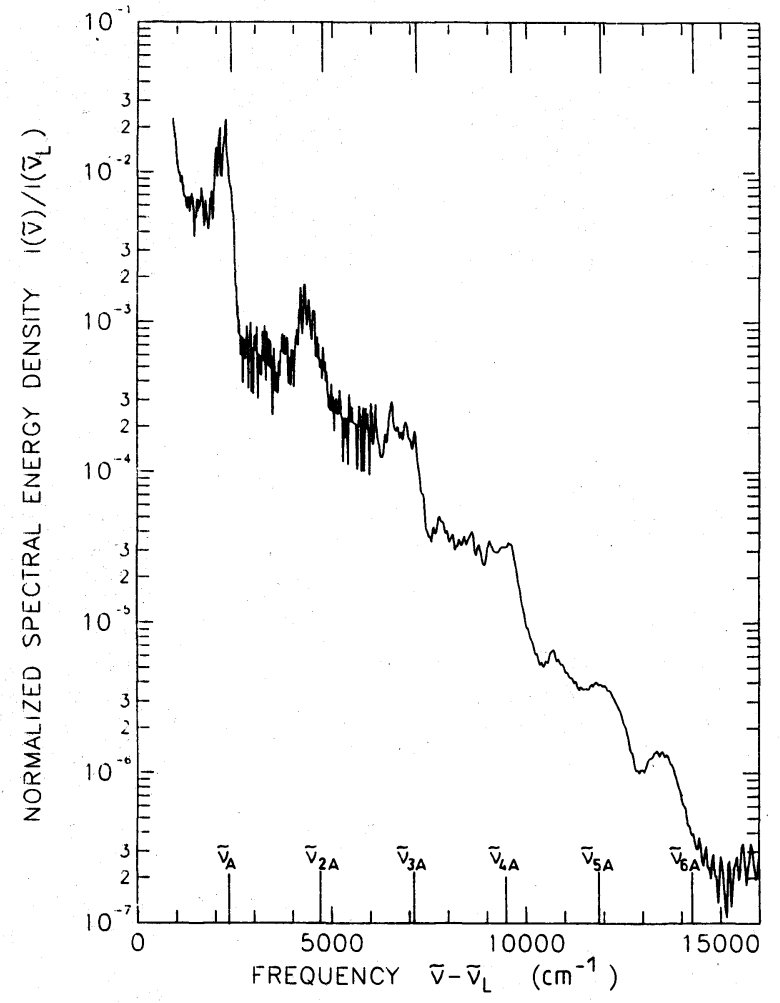

Figure 4 Output spectrum behind $\mathrm{D}_{2} \mathrm{O}$ sample. Fundamental laser excitation. Connection of spectra of SP1 and SP2 at $\tilde{\nu}-$ $\tilde{\nu}_{L}=6000 \mathrm{~cm}^{-1} \cdot W_{L, \text { in }} \approx 90 \mu \mathrm{J} . T_{c} \approx 0.15$.

generated continuum decreases rapidly for $\tilde{\nu}<\tilde{\nu}_{R}$. At the antiStokes side in the range between $\tilde{\nu}_{L}=\tilde{\nu}_{L}+\delta \tilde{\nu}_{R}$ and $\tilde{\nu}_{L}+2 \delta \tilde{\nu}_{R}$ some signal enhancement due to Stokes Ramaninitiated antiStokes up-conversion is observable. The strongly fluctuating signal at low spectral energy density is due to the noisy behaviour of the detection systems at their detection limits.

The spectrum of Fig. 9 was obtained by spatially blocking the central part of the forward emission to spectrometer SP2 (frequency range $\tilde{\nu}-\tilde{\nu}_{S H}>-3200 \mathrm{~cm}^{-1}$; spectral region of $\tilde{\nu}-\tilde{\nu}_{S H}<-3200 \mathrm{~cm}^{-1}$ is detected with spectrometer SP1). The light emitted in a cone of $\theta \gtrsim 0.025 \mathrm{rad}$ was collected ( $\theta$ is full cone angle). The input pulse energy was $W_{S H, \text { in }} \approx 450 \mu \mathrm{J}$ and the central spectral transmission was $T_{c} \approx 0.25$. At the longwavelength side the stimulated second Stokes component is observed and at the shortwavelength side antiStokes up-conversion of light from the first antiStokes region to the second antiStokes region is observed.

The dashed curve in Fig. 8 shows the output pulse spectrum of a pulse of input energy $W_{S H, \text { in }}=680 \mu \mathrm{J}$ focused to the $\mathrm{D}_{2} \mathrm{O}$ sample with a lens of focal length $f=80 \mathrm{~cm}$. The transmission of the central spectral part was $T_{c}=0.74$. The spectral features are similar to the solid curve in Fig. 8. Only the efficiency of stimulated first Stokes Raman light generation is somewhat reduced and no coherent antiStokes Raman feature is resolved.

In the experiments with the 80 -cm focusing lens the peak intensity of the input secondharmonic light was determined by two-photon energy transmission measurement through a rutile crystal (photodetectors PD1 and PD2) [72]. The threshold intensity of efficient stimu- 


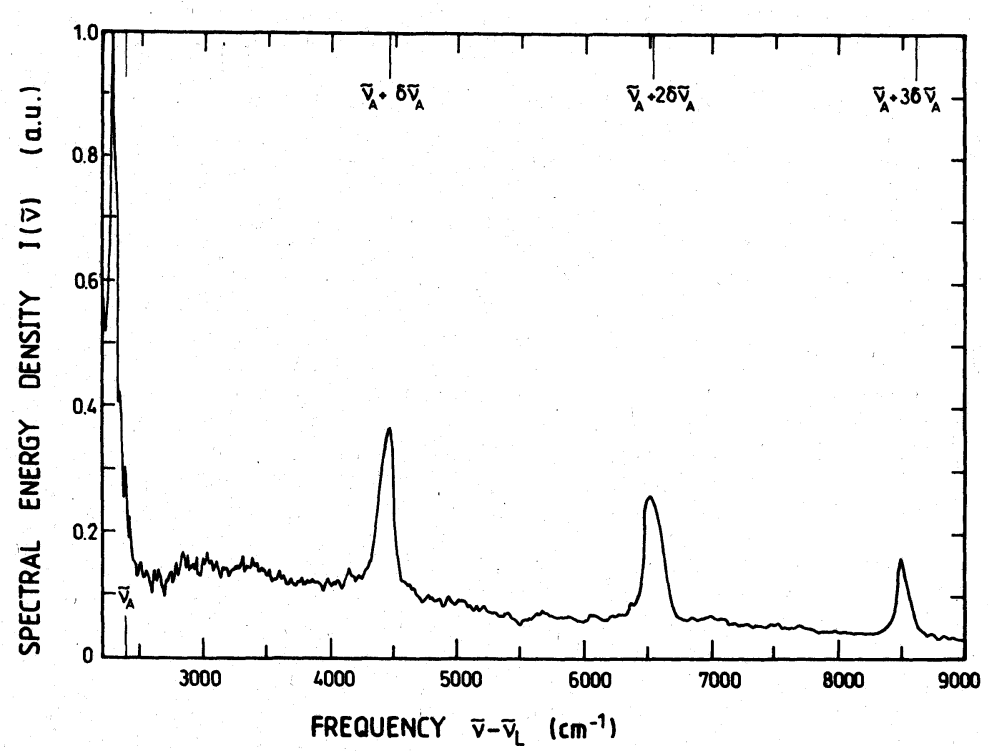

Figure 5 Multiple-peaked output spectrum behind $\mathrm{D}_{2} \mathrm{O}$ sample obtained once in a series of about 300 shots. Fundamental laser excitation. Only spectrometer SP1 was used in recording.

lated Raman scattering was found to be $I_{S H, \text { th }}^{(R)} \approx 1.4 \times 10^{10} \mathrm{~W} \mathrm{~cm}^{-2}$. This threshold intensity is lower than expected theoretically (see below), indicating that self-focusing ircreases the intensity in the sample even for input pulse focusing with a lens of $80 \mathrm{~cm}$ focal length.

Using a 25-cm focusing lens stimulated Raman light generation and continuum light generation were observed for input pump energies down to about $5 \mu \mathrm{J}$. At the threshold of continuum and Raman light observation the relative magnitude of Raman light to continuum light changed from shot to shot. Figure 10 depicts three examples illustrating the variation of the spectral shapes of the generated light. The spectral region around the pump laser frequency was blocked by filters and is not displayed. In Fig. 10a the parametric continuum generation dominates. In Fig. 10b the spectrum shows stimulated Raman light, coherent antiStokes Raman light, and pump-pulse-nondegenerate parametric four-photon light (interaction process $\tilde{\nu}_{S H}+\tilde{\nu}_{R} \rightarrow \tilde{\nu}_{3^{\prime}}+\tilde{\nu}_{4^{\prime}}$, strong peak at $\tilde{\nu}-\tilde{\nu}_{L} \approx 1850 \mathrm{~cm}^{-1}$ and strong signal at $\tilde{\nu}-\tilde{\nu}_{L}=600 \mathrm{~cm}^{-1}$ ). The spectrum in Fig. $10 \mathrm{c}$ reveals only stimulated Stokes Raman scattering.

The radial distribution of the output emission in some frequency regions is displayed in Fig. 11 (photodiode-array detector $11 \mathrm{~cm}$ behind $\mathrm{D}_{2} \mathrm{O}$ sample). The focal length of the input focusing lens $\mathrm{L} 1$ was $f=25 \mathrm{~cm}$. The cross-sectional distribution of an input pump pulse is shown in Fig. 11a. Its divergence angle is $\Delta \theta \approx 0.017$ (FWHM). At the shortwavelength side the beam divergence increases from $500 \mathrm{~nm}$ (Fig. 11b) to $470 \mathrm{~nm}$ (Fig. 11c). The radial distribution of Fig. 11c includes the coherent antiStokes Raman scattering. At the long-wavelength side the beam divergence increases from $550 \mathrm{~nm}$ (Fig. 11d) to $590 \mathrm{~nm}$ (Fig. 11e). The radial distribution of Fig. 11f $(\lambda=599.4 \mathrm{~nm} \pm 9 \mathrm{~nm})$ is dominated by the stimulated Stokes Raman light. Its beam diameter is reduced compared to the beam extensions of Figs $11 \mathrm{~b}$ to $11 \mathrm{e}$. The measured average beam divergences (half-width at half- 


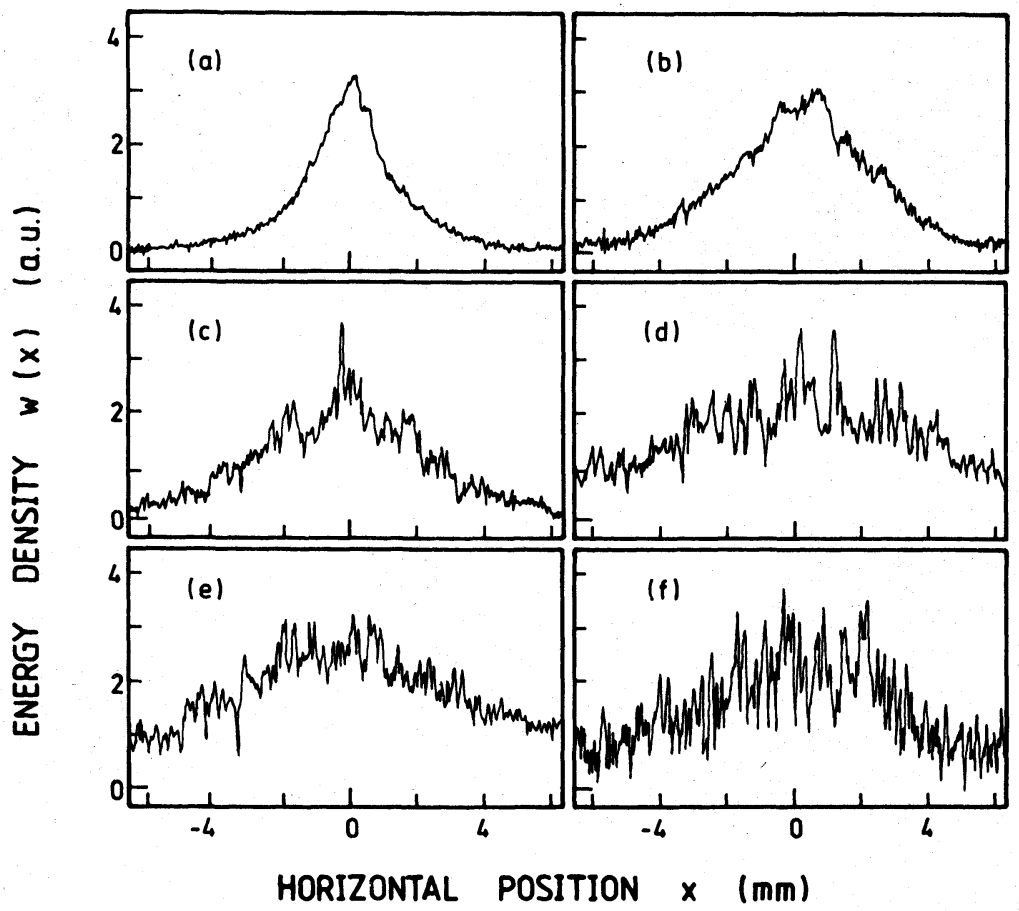

Figure 6 Spatial distribution of spectral parts of superbroadened output pulses $12 \mathrm{~cm}$ behind $\mathrm{D}_{2} \mathrm{O}$ sample. Input laser wavelength is $\lambda_{L}=1054 \mathrm{~nm}$. (a) Wavelength $\lambda=1054 \mathrm{~nm}$. Spectral half-width of interference filter $\Delta \lambda_{l F}=7.6 \mathrm{~nm}$ (FWHM). Input pulse energy $W_{L, \text { in }}=850 \mu \mathrm{J}$. (b) $\lambda \approx 1150 \mathrm{~nm}$ (long-wavelength passing filter opening at $1150 \mathrm{~nm}$ ), $W_{L l, \text { in }}=500 \mu \mathrm{J}$. (c ) $\lambda=667 \mathrm{~nm}, \Delta \lambda_{l F}=8.4 \mathrm{~nm}, W_{L, \text { in }}=700 \mu \mathrm{J}$. (d) $\lambda=500 \mathrm{~nm}, \Delta \lambda_{l F}=11 \mathrm{~nm}, W_{L, \text { in }}=1100 \mu \mathrm{J}$. (e) $\lambda=470 \mathrm{~nm}, \Delta \lambda_{l F}=9.4 \mathrm{~nm}, W_{L, \text { in }}=1300 \mu \mathrm{J}$. (f) $\lambda=405.4 \mathrm{~nm}, \Delta \lambda_{\text {IF }}=10.5 \mathrm{~nm}, W_{L \text {, in }}=850 \mu \mathrm{J}$. Noisy signal appearance is thought to be due to noisy behaviour of photodiode array detector.

maximum) are displayed as triangles in Fig. 15. Conical rainbow-like emission was not observed.

\section{Discussion}

The experimental observations are interpreted by the interaction of collinear non-phasematched parametric four-photon interaction, longitudinally phase-matched, transversally non-phase-matched (Čerenkov-like) stimulated parametric four-photon interaction under self-focusing conditions, stimulated Raman scattering, longitudinally phase-matched coherent antiStokes Raman scattering, inverse Raman scattering, self-phase modulation and cross-phase modulation-induced collinear phase-matched stimulated parametric fourphoton interaction in the frequency range between the pump laser frequency and the first Stokes frequency, and Raman-induced cascading light up-conversion. The energy diagrams and wavevector diagrams of the various processes are sketched in Fig. 12. They are described theoretically and their order of magnitude efficiencies are estimated in the following.

4.1. General equation system of pump-pulse-degenerate parametric fourphoton interaction including Raman scattering and phase-modulation

A general wave equation system in the slowly varying moving-frame approximation 


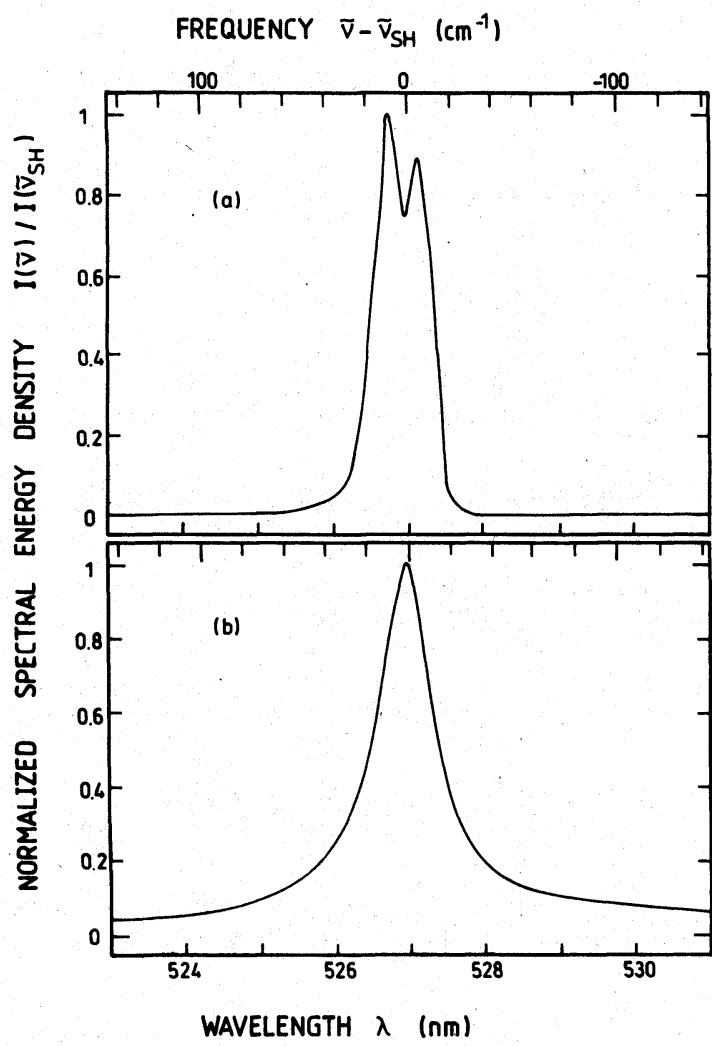

Figure 7 Spectra of second harmonic light pulses: (a) without passing through $\mathrm{D}_{2} \mathrm{O}$ sample (pulse energy $W_{L, \text { in }}=440 \mu \mathrm{J}$ ); (b) passing through $D_{2} O$ sample $\left(W_{L, \text { in }}=630 \mu \mathrm{J}\right)$.

including stimulated Raman scattering $\left(\omega_{1} \rightarrow \omega_{4}+\omega_{v} ; \omega_{1}=\omega_{L}\right.$ or $\omega_{S H}, \omega_{4}=\omega_{R}=\omega_{1}-\omega_{v}$, coupling nonlinear susceptibility $\left.\chi_{R}^{\prime \prime}=\chi^{(3)^{\prime \prime}}\left(-\omega_{4} ; \omega_{1},-\omega_{1}, \omega_{4}\right)\right)$, inverse Raman scattering $\left(\omega_{3} \rightarrow \omega_{1}+\omega_{v} ; \omega_{3}=\omega_{A}, \chi_{A}^{\prime \prime}=\chi^{(3)^{\prime \prime}}\left(-\omega_{3} ; \omega_{1},-\omega_{1}, \omega_{3}\right) \approx-\chi_{R}^{\prime \prime}\right)$, pump-pulsedegenerate parameteric four-photon interaction $\left(\omega_{1}+\omega_{1} \rightarrow \omega_{3}+\omega_{4} ; \chi^{(3)}\left(-\omega_{3} ; \omega_{1}, \omega_{1}\right.\right.$, $\left.-\omega_{4}\right)$ with Stokes-antiStokes Raman coupling resonance structure) as well as self-phase modulation $\left(\omega_{1}+\omega_{1} \rightarrow \omega_{1}+\omega_{1}, \chi^{(3)^{\prime}}\left(-\omega_{1} ; \omega_{1},-\omega_{1}, \omega_{1}\right)\right)$ and cross-phase modulation (e.g. $\left.\omega_{1}+\omega_{4} \rightarrow \omega_{1}+\omega_{4}, \chi^{(3)^{\prime}}\left(-\omega_{4} ; \omega_{1},-\omega_{1}, \omega_{4}\right)\right)$ reads [51, 58, 63]:

$$
\begin{aligned}
\frac{\partial E_{3}}{\partial z}+\frac{\alpha_{3}}{2} E_{3}= & -\mathrm{i} \frac{\omega_{3}}{2 n_{3} c_{0}}\left\{3 \chi^{(3)}\left(-\omega_{3} ; \omega_{3},-\omega_{3}, \omega_{3}\right)\left|E_{3}\right|^{3} E_{3}\right. \\
& +6 \chi^{(3)^{\prime}}\left(-\omega_{3} ; \omega_{1},-\omega_{1}, \omega_{3}\right)\left|E_{1}\right|^{2} E_{3}+6 \chi^{(3)}\left(-\omega_{3} ; \omega_{4},-\omega_{4}, \omega_{3}\right)\left|E_{4}\right|^{2} E_{3} \\
& +3 \chi^{(3)}\left(-\omega_{3} ; \omega_{1}, \omega_{1},-\omega_{4}\right) E_{1}^{2} E_{4}^{*} \exp (\mathrm{i} \Delta \mathbf{k r}) \\
& \left.-\mathrm{i} 6 \chi^{(3)^{\prime \prime}}\left(-\omega_{3} ; \omega_{1},-\omega_{1}, \omega_{3}\right)\left|E_{1}\right|^{2} E_{3}\right\}
\end{aligned}
$$

$$
\begin{aligned}
\frac{\partial E_{4}}{\partial z}+\frac{\alpha_{4}}{2} E_{4}= & -\mathrm{i} \frac{\omega_{4}}{2 n_{4} c_{0}}\left\{3 \chi^{(3)}\left(-\omega_{4} ; \omega_{4},-\omega_{4}, \omega_{4}\right)\left|E_{4}\right|^{2} E_{4}\right. \\
& +6 \chi^{(3)^{\prime}}\left(-\omega_{4} ; \omega_{1},-\omega_{1}, \omega_{4}\right)\left|E_{1}\right|^{2} E_{4}+6 \chi^{(3)}\left(-\omega_{4} ; \omega_{3},-\omega_{3}, \omega_{4}\right)\left|E_{3}\right|^{2} E_{4}
\end{aligned}
$$




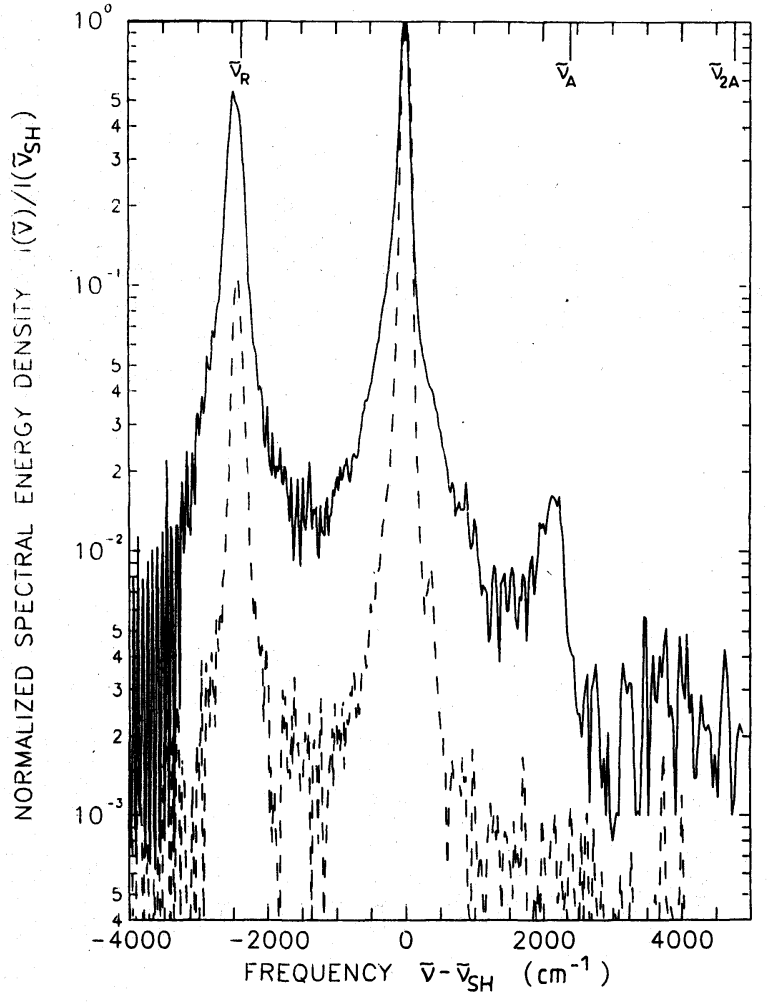

Figure 8 Spectral distribution of pulse behind $\mathrm{D}_{2} \mathrm{O}$ sample. Second harmonic laser excitation (spectrum detected with spectrometer SP2). Solid curve: focal length of lens $L 1$ is $f=25 \mathrm{~cm}$. Input pulse energy $W_{L, \text { in }} \approx 750 \mu \mathrm{J}$. Central spectral pulse transmission $T_{c} \approx 0.24$. Dashed curve: $f=80 \mathrm{~cm}, W_{L, \text { in }} \approx 680 \mu \mathrm{J} . T_{c} \approx 0.74$. Input peak intensity $I_{0 L} \approx 3 \times 10^{10} \mathrm{~W} \mathrm{~cm}^{-2}$.

$$
\begin{aligned}
& +3 \chi^{(3)}\left(-\omega_{4} ; \omega_{1}, \omega_{1},-\omega_{3}\right) E_{1}^{2} E_{3}^{*} \exp (\mathrm{i} \Delta \mathbf{k r}) \\
& \left.-\mathrm{i} 6 \chi^{(3)^{\prime \prime}}\left(-\omega_{4} ; \omega_{1},-\omega_{1}, \omega_{4}\right)\left|E_{1}\right|^{2} E_{4}\right\}
\end{aligned}
$$

$$
\begin{aligned}
\frac{\partial E_{1}}{\partial z}+\frac{\alpha_{1}}{2} E_{1}= & -\mathrm{i} \frac{\omega_{1}}{2 n_{1} c_{0}}\left\{3 \chi^{(3)}\left(-\omega_{1} ; \omega_{1},-\omega_{1}, \omega_{1}\right)\left|E_{1}\right|^{2} E_{1}\right. \\
& +6 \chi^{(3)^{\prime}}\left(-\omega_{1} ; \omega_{3},-\omega_{3}, \omega_{1}\right)\left|E_{3}\right|^{2} E_{1}+6 \chi^{(3)^{\prime}}\left(-\omega_{1} ; \omega_{4},-\omega_{4}, \omega_{1}\right)\left|E_{4}\right|^{2} E_{1} \\
& +6 \chi^{(3)}\left(-\omega_{1} ; \omega_{3}, \omega_{4},-\omega_{1}\right) E_{1}^{*} E_{3} E_{4} \exp (-\mathrm{i} \Delta \mathbf{k r})\left|E_{3}\right|^{2} E_{1} \\
& \left.-\mathrm{i} 6 \chi^{(3)^{\prime \prime}}\left(-\omega_{1} ; \omega_{3},-\omega_{3}, \omega_{1}\right)\left|E_{3}\right|^{2} E_{1}-\mathrm{i} 6 \chi^{(3)^{\prime \prime}}\left(-\omega_{1} ; \omega_{4},-\omega_{4}, \omega_{1}\right)\left|E_{4}\right|^{2} E_{1}\right\}
\end{aligned}
$$

The $E_{i}(i=1,3,4 ; 1=\mathrm{L}$ or $\mathrm{SH})$ are the amplitudes of the electric field strengths; the $\alpha_{i}$ and $n_{i}$ are the absorption coefficients and refractive indices at the angular frequencies $\omega_{i}$, respectively; $c_{0}$ is the vacuum light velocity. The first terms in the equations describe selfphase modulation (and two-photon absorption in the case of complex nonlinear susceptibility $\chi^{(3)}=\chi^{(3)^{\prime}}-\mathrm{i} \chi^{(3)^{\prime \prime}}$; two-photon absorption does not occur here since there is no excited state at $\left.2 \omega_{i}\right)$. The second and third terms present cross-phase modulation. The fourth terms are responsible for pump-pulse-degenerate parametric four-photon interaction. The last term of Equation 1 takes care of inverse Raman scattering. In Equation 2 it relates to stimulated Raman scattering. The last two terms of Equation 3 describe 


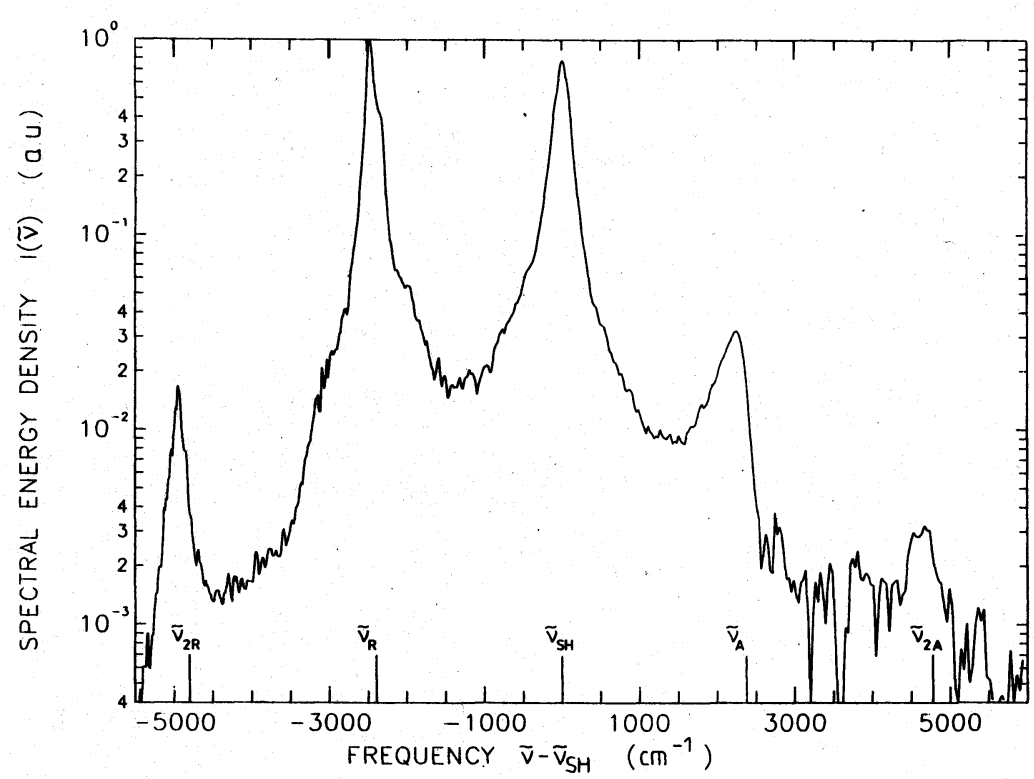

Figure 9 Spectral distribution of a pulse behind $\mathrm{D}_{2} \mathrm{O}$ sample. Central spatial part was covered with a black tape spot of $1 \mathrm{~cm}$ diameter for light in spectral range $\tilde{\nu}-\tilde{\nu}_{S H}>-3200 \mathrm{~cm}^{-1}$ (full cone angle $\theta \approx 2.5 \times 10^{-2}$ ). Input pulse energy $W_{L, \text { in }} \approx 630 \mu \mathrm{J}$. Central spectral pulse transmission $T_{c} \approx 0.24$.

inverse Raman scattering and stimulated Raman scattering, respectively. $\Delta k=k_{3}+k_{4}-$ $2 \mathbf{k}_{1}$ is the wavevector mismatch; $\mathbf{r}$ is the position vector of $\mathbf{E}$. The factors in front of the nonlinear susceptibilities are the frequency-degeneracy factors $D$ (in the case of $\chi^{(3)}\left(-\omega_{i} ; \omega_{j}, \omega_{k}, \omega_{l}\right)$ it is $D=1$ for $\omega_{j}=\omega_{k}=\omega_{l}, D=3$ for two equal frequencies of $\omega_{j}, \omega_{k}, \omega_{l}$, and $D=6$ when all three frequencies $\omega_{j}, \omega_{k}$ and $\omega_{l}$ are different).

The secondary pump-pulse-nondegenerate parametric four-photon amplification process $\omega_{1}+\omega_{R} \rightarrow \omega_{3^{\prime}}+\omega_{4^{\prime}}$ ( $\omega_{R}$ frequency of first Stokes Raman light) and the cascading up-conversion processes $\omega_{1}+\omega_{(i)}-\omega_{R} \rightarrow \omega_{(i+1)}$ are not included in the equation system 13. They are separately discussed below in Sections 4.6 and 4.7.

The absorption coefficient spectrum $\alpha(\tilde{\nu})$ of $\mathrm{D}_{2} \mathrm{O}$ at room temperature is displayed in Fig. 13 (curve 1 represents our own measurements of our $\mathrm{D}_{2} \mathrm{O}$ sample; curves 2 and 3 are data taken from $[73,74]$ ). Over a wide frequency range (from $\tilde{\nu} \approx 6000 \mathrm{~cm}^{-1}$ to the UV region) the absorption is negligible. In our discussion the absorption coefficients are neglected. In regions of high absorption the third-order nonlinear susceptibilities are resonantly enhanced [23]. Absorptive nonlinear susceptibility enhancements are not taken into consideration here.

The refractive index spectrum of $\mathrm{D}_{2} \mathrm{O}$ at room temperature is included in Fig. 13 (curve $n$, data taken from $[75,76]$ and interpolation). The refractive index dispersion determines the phase-matching situation of the parametric four-photon interaction processes.

In the case of collinear interaction the wavevector mismatch for the process $\omega_{1}+\omega_{2} \rightarrow$ $\omega_{3}+\omega_{4}$ is given by

$$
\Delta k=k_{3}+k_{4}-k_{1}-k_{2}=2 \pi\left(n_{3} \tilde{\nu}_{3}+n_{4} \tilde{\nu}_{4}-n_{1} \tilde{\nu}_{1}-n_{2} \tilde{\nu}_{2}\right)
$$

In Fig. 14 the pump-pulse-degenerate collinear wavevector-mismatch $\Delta k\left(\omega_{1}+\omega_{1} \rightarrow\right.$ $\left.\omega_{3}+\omega_{4}\right)$ (solid curves) and the pump-pulse-nondegenerate collinear wavevector mismatch 


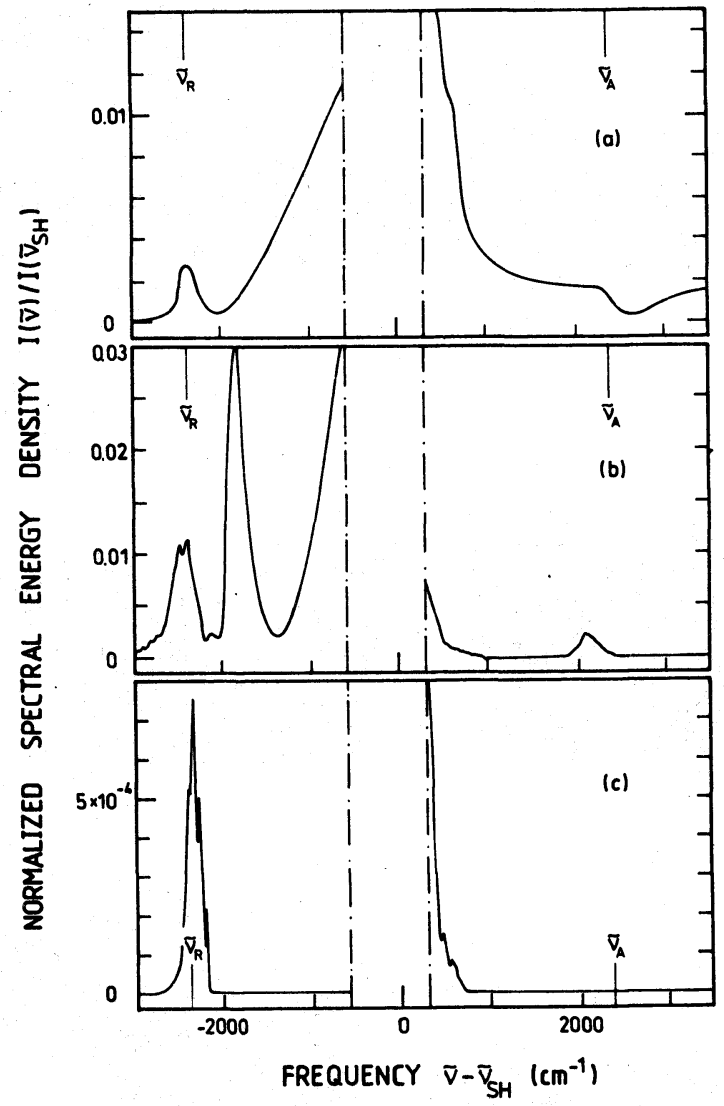

Figure 10 Examples of output spectra behind $\mathrm{D}_{2} \mathrm{O}$ sample for low input pulse energies. Absolute scale of ordinates is only correct within a factor of 2. (a) Input pulse energy $W_{L, \text { in }} \approx 8 \mu \mathrm{J}$. (b) $W_{L, \text { in }} \approx 24 \mu \mathrm{J}$. (c) $W_{L, \text { in }} \approx$ $16 \mu \mathrm{J}$.

$\Delta k\left(\omega_{1}+\omega_{R} \rightarrow \omega_{3^{\prime}}+\omega_{4^{\prime}}\right) \quad$ (long-dashed curves) are shown for $\lambda_{1}=2 \pi /\left(c_{0} \omega_{1}\right)=$ $\lambda_{L}=1.054 \mu \mathrm{m}, \lambda_{1}=\lambda_{S H}=527 \mathrm{~nm}$, and $\omega_{R}=\omega_{1}-\omega_{v}\left(\tilde{\nu}_{v}=\omega_{v} /\left(2 \pi c_{0}\right)=2380 \mathrm{~cm}^{-1}\right)$. In the nondegenerate case the wavevector mismatch between $\tilde{\nu}_{1}$ and $\tilde{\nu}_{2}=\tilde{\nu}_{R}$ is negative and intensity-dependent collinear phase-matching becomes possible from nonlinear refractive index contributions $[51,58,63,77]$.

The noncollinear internal phase-matching angle $\varphi_{3 P}$ (angle between $\mathbf{k}_{1}$ and $\mathbf{k}_{3}$ ) of the degenerate interaction $\omega_{1}+\omega_{1} \rightarrow \omega_{3}+\omega_{4}$ is given by (trigonometric cosine law)

$$
\varphi_{3 P}=\arccos \left[\frac{4 k_{1}^{2}+k_{3}^{2}-k_{4}^{2}}{2 k_{3} k_{4}}\right]=\arccos \left[\frac{4 n_{1}^{2} \tilde{\nu}_{1}^{2}+n_{3}^{2} \tilde{\nu}_{3}^{2}-n_{4}^{2} \tilde{\nu}_{4}^{2}}{2 n_{3} n_{4} \tilde{\nu}_{3} \tilde{\nu}_{4}}\right]
$$

The solid curves in Fig. 15 display $\varphi_{P}(\tilde{\nu})$ in $\mathrm{D}_{2} \mathrm{O}$ for $\lambda_{L}=1054 \mathrm{~nm}$ and $\lambda_{S H}=527 \mathrm{~nm}$.

The phase-matching relation $\Delta \mathbf{k r}=\Delta k_{x} x+\Delta k_{y} y+\Delta k_{z} z=0$ generally requires $\Delta k_{x}=\Delta k_{y}=\Delta k_{z}=0$. Under self-focusing or self-trapping conditions [64] it relaxes to $\Delta k_{z}=0$ ( $z$ is the propagation direction, longitudinal phase-matching) since the transversal phases reduce to $\Delta k_{x} x=\Delta k_{\mathrm{tr}} x \leq \Delta k_{\mathrm{tr}} d<\pi$ and $\Delta k_{y} y=\Delta k_{\mathrm{tr}} y \leq \Delta k_{\mathrm{tr}} d<\pi$ for sufficiently small filament diameters $d$. The longitudinally phase-matched $\left(\Delta k_{z}=0\right)$ and transversally non-phase-matched $\left(\Delta k_{\mathrm{tr}} \neq 0\right)$ parametric four-photon interaction resembles the off-axis Cerenkov radiation [41, 78-82] and the Cerenkov-like second- 


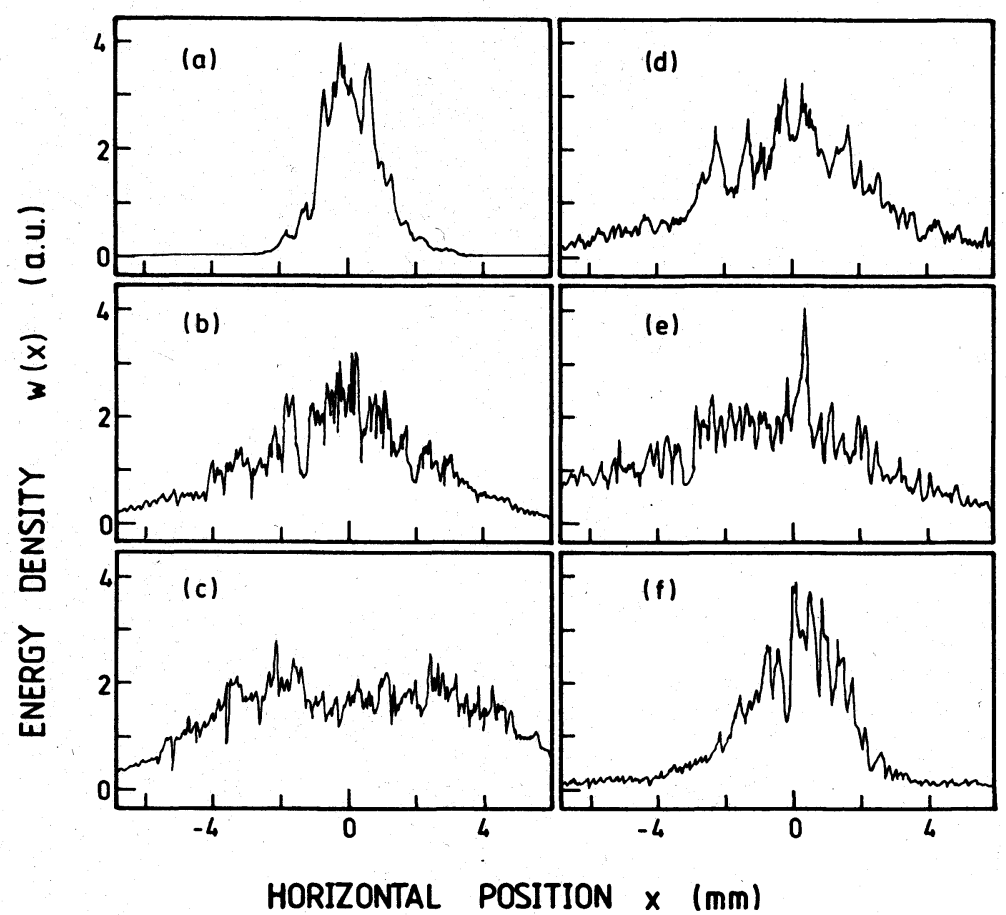

Figure 11 Spatial distribution of spectral parts of superbroadened output pulses $11 \mathrm{~cm}$ behind $\mathrm{D}_{2} \mathrm{O}$ sample. Input pulse wavelength is $\lambda_{S H}=527 \mathrm{~nm}$. (a) Wavelength $\lambda=\lambda_{S H}$. Without $\mathrm{D}_{2} O$. (b) $\lambda=500 \mathrm{~nm}$. Spectral half-width of interference filter $\Delta \lambda_{I F}=4.5 \mathrm{~nm}$ (FWHM). Input pulse energy $W_{L, \text { in }} \approx 440 \mu \mathrm{J}$. (c) $\lambda=470 \mathrm{~nm}$, $\Delta \lambda_{\text {IF }}=9.4 \mathrm{~nm}, W_{L, \text { in }} \approx 750 \mu \mathrm{J}$. (d) $\lambda=550 \mathrm{~nm}, \Delta \lambda_{\text {IF }}=10.5 \mathrm{~nm}, W_{L, \text { in }} \approx 190 \mu \mathrm{J}$. (e) $\lambda=590 \mathrm{~nm}, \Delta \lambda_{\text {IF }}=$ $10 \mathrm{~nm}$. $W_{L \text {, in }} \approx 500 \mu \mathrm{J}$. (f) $\lambda=599.4 \mathrm{~nm}, \Delta \lambda_{l F}=9 \mathrm{~nm}, W_{L, \text { in }} \approx 310 \mu \mathrm{J}$.

harmonic generation in light guides [83-85], and may be called Čerenkov-like parametric four-photon interaction.

The longitudinal phase-matching condition $\Delta k_{z}=0$ of the process $\omega_{1}+\omega_{1} \rightarrow \omega_{3}+\omega_{4}$ reads (Fig. 12)

$$
\begin{aligned}
\Delta k_{z} & =k_{3} \cos \varphi_{3}+k_{4} \cos \varphi_{4}-2 k_{1} \\
& =2 \pi\left(n_{3} \tilde{\nu}_{3} \cos \varphi_{3}+n_{4} \tilde{\nu}_{4} \cos \varphi_{4}-2 n_{1} \tilde{\nu}_{1}\right)=0
\end{aligned}
$$

Longitudinal phase-matching is achieved for corresponding internal $\left(\varphi_{3}, \varphi_{4}\right)$ angle pairs in the region $0 \leq \varphi_{3} \leq \varphi_{3 c}$ and $\varphi_{4 c} \geq \varphi_{4} \geq 0\left(\varphi_{3}=0\right.$ corresponds to $\varphi_{4}=\varphi_{4 c}$, and $\varphi_{3}=\varphi_{3 c}$ corresponds to $\varphi_{4}=0$ ). This angle range is responsible for longitudinally phase-matched parametric light emission in the forward direction with a bell-shaped radial intensity profile. The maximum longitudinal phase-matching angle $\varphi_{c}$ versus $\tilde{\nu}$ is displayed by the dashed curves in Fig. 15 for $\lambda_{1}=1054 \mathrm{~nm}$ and $527 \mathrm{~nm}$. The maximum transversal wavevector mismatch $\Delta k_{\mathrm{tr}, \mathrm{m}}$ for $\varphi=\varphi_{c}\left(\Delta k_{\mathrm{tr}, \mathrm{m}}=k_{i} \sin \varphi_{i c}, i=3,4\right)$ is displayed by the shortdashed curves in Fig. 14. For $\varphi=\varphi_{c}$ the condition $\Delta k_{\text {tr }} d<\pi$ is fulfilled over the complete displayed range for $\lambda_{1}=\lambda_{L}=1054 \mathrm{~nm}$ and in the frequency range $16000 \mathrm{~cm}^{-1}<\tilde{\nu}<21500 \mathrm{~cm}^{-1}$ for $\lambda_{1}=\lambda_{S H}=527 \mathrm{~nm}$ if $d=5 \mu \mathrm{m}$ (typical filament diameter [64]). 
(a)

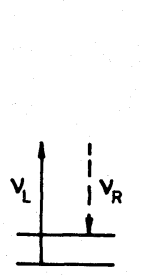

(b)

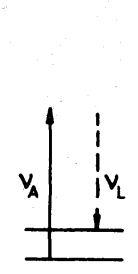

(c)

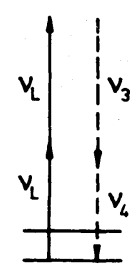

(d)

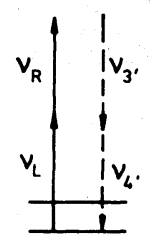

(e)

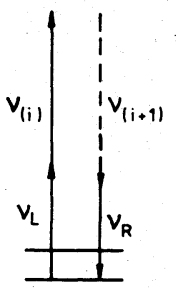

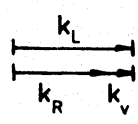
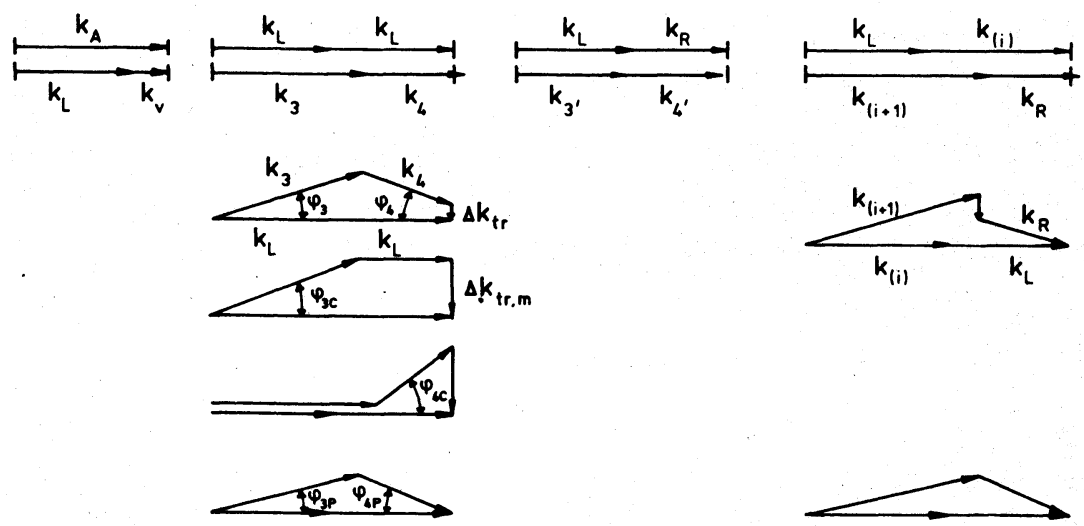

Figure 12 Energy level diagrams and wavevector diagrams of various involved interaction processes. (a) Stimulated Raman scattering. (b) Inverse Raman scattering. (c) Pump-pulse-degenerate stimulated parametric four-photon interaction, or coherent antiStokes Raman scattering (CARS). (d) Pump-pulsenondegenerate stimulated parametric four-photon interaction (no input signal) or nondegenerate parametric four-photon amplification (with input signal). (e) Cascading light up-conversion. First row: energy level diagrams. Second row: collinear wavevector diagrams. Third to fifth row: longitudinally phase-matched wavevector diagrams. Sixth row: noncollinearly phase-matched wavevector diagrams.

The noncollinear parametric interaction reduces the spatial overlap $l_{i u}=d / \tan \varphi$ of parametric light and pump light. In Fig. 16 overlap ratios $l_{u} / d$ are displayed for the degenerate four-photon interaction $\omega_{1}+\omega_{1} \rightarrow \omega_{3}+\omega_{4}$. The solid curves apply to noncollinear phasematched interaction, while the dashed curves belong to longitudinally phase-matched interaction where the minimal overlap ratios $l_{u, c}=d / \tan \varphi_{c}$ are presented.

The third-order nonlinear susceptibilities involved in the equation system 1-3 are approximated in the following. Only nonresonant and Raman-type (difference frequency) resonance contributions are retained. The susceptibilities $\chi^{(3)}\left(-\omega_{i} ; \omega_{i},-\omega_{i}, \omega_{i}\right)$ $(i=\mathrm{L}, \mathrm{SH}, 3,4)$ are set equal to the real parts $\chi^{(3)^{\prime}}\left(-\dot{\omega}_{i} ; \omega_{i},-\omega_{i}, \omega_{i}\right)$ which are responsible for self-phase modulation. They are related to the field coefficients $n_{2, \text { nl }}$ of the nonlinear refractive index by [86]

$$
\chi^{(3)^{\prime}}\left(-\omega_{i} ; \omega_{i},-\omega_{i}, \omega_{i}\right)=\left(n_{i} / 3\right) n_{2, \mathrm{nl}}\left(\omega_{i}, \omega_{i}\right)
$$

Their values for different $i(i=\mathrm{L}, \mathrm{SH}, 3,4)$ are set equal, i.e. $\chi^{(3)^{\prime}}\left(-\omega_{i} ; \omega_{i},-\omega_{i}, \omega_{i}\right)=\chi_{\mathrm{nl}}^{(3)^{\prime}}$. $n_{2, \mathrm{nl}}(527 \mathrm{~nm}, 527 \mathrm{~nm})=2.2 \times 10^{-22} \mathrm{~m}^{2} \mathrm{~V}^{-2}$ is reported in [22], giving $\chi_{\mathrm{nl}}^{(3)^{\prime}}=9.8 \times 10^{-23}$ $\mathrm{m}^{2} \mathrm{~V}^{-2}$. The susceptibilities $\chi^{(3)^{\prime}}\left(-\omega_{i} ; \omega_{j},-\omega_{j}, \omega_{i}\right)(i \neq j)$ which are responsible for crossphase modulation are approximately equal to $\chi^{(3)^{\prime}}\left(-\omega_{i} ; \omega_{i},-\omega_{i}, \omega_{i}\right)=\chi_{\mathrm{nl}}^{(3)^{\prime}}$ (Raman-type 


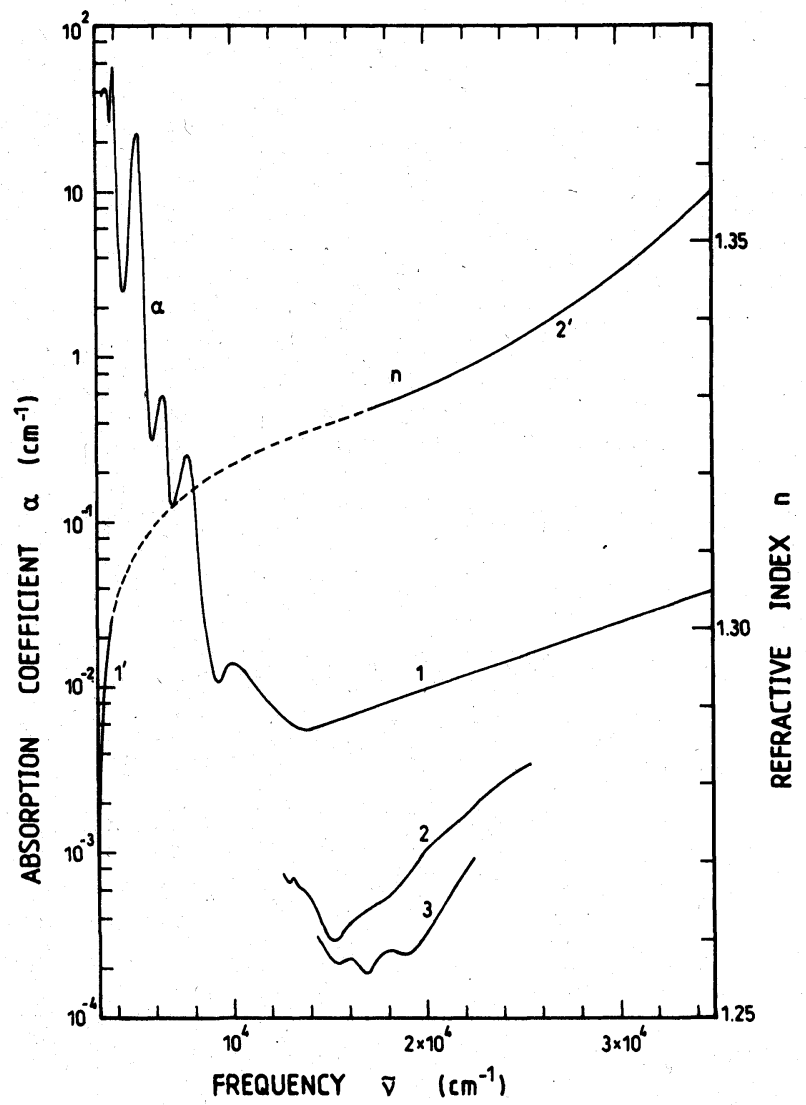

Figure 13 Absorption coefficient spectrum $\alpha(\tilde{\nu})$ and refractive index spectrum $n(\tilde{\nu})$ of $D_{2} 0$ at room temperature. Absorption curves: 1 , own measurements; 2 , taken from [73]; 3, taken from [74]. Refractive index curve: part 1, taken from [75]; part 2 taken from [76]; dashed part, interpolated.

contribution to real part of nonlinear susceptibility is zero at exact resonance). The nonlinear refractive index coefficient responsible for cross-phase modulation is $n_{2, \text { nl }}\left(\omega_{i}, \omega_{j}\right)=$ $2 n_{2, \mathrm{nl}}\left(\omega_{i}, \omega_{i}\right)$.

The stimulated Raman scattering susceptibility and inverse Raman scattering susceptibility are given by [54]

$$
\chi^{(3)^{\prime \prime}}\left(-\omega_{4} ; \omega_{1},-\omega_{1}, \omega_{4}\right) \approx-\sum_{m} \chi_{R, m}^{\prime \prime} \frac{\Gamma_{m}^{2}}{\left(\omega_{v, m}-\omega_{1}+\omega_{4}\right)^{2}+\Gamma_{m}^{2}}
$$

and

$$
\chi^{(3)^{\prime \prime}}\left(-\omega_{3} ; \omega_{1},-\omega_{1}, \omega_{3}\right) \approx \sum_{m} \chi_{R, m}^{\prime \prime} \frac{\Gamma_{m}^{2}}{\left(\omega_{1, m}-\omega_{3}+\omega_{1}\right)^{2}+\Gamma_{m}^{2}}
$$

respectively: $m$ runs over the various vibrational modes. $\Gamma_{m}$ is half the spectral width at halfheight of the Raman transition $m$ (half the spectral width at half-height of the spontaneous cmission) and $\backslash_{k, m}^{\prime \prime}$ is the peak Raman susceptibility of the $m$ th vibrational mode of 


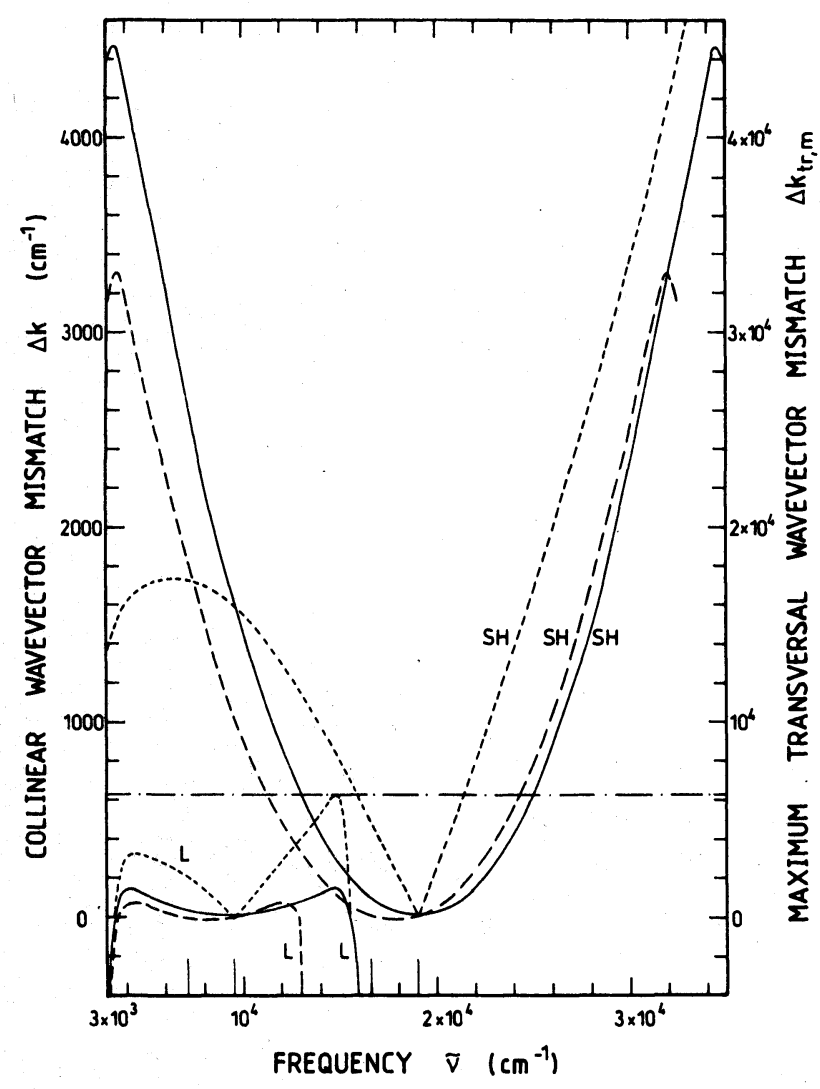

Figure 14 Wavevector mismatch for various interaction processes. Solid curves, collinear wavevector mismatch for interaction $\omega_{1}+\omega_{1} \rightarrow \omega_{3}+\omega_{4}$. Long-dashed curves, collinear wavevector mismatch for interaction $\omega_{1}+\omega_{R} \rightarrow \omega_{3^{\prime}}+\omega_{4^{\prime}}$. Short-dashed curves (right ordinate), maximum transversal wavevector mismatch (signal or idler light propagate parallel to pump light) for the interaction $\omega_{1}+\omega_{1} \rightarrow \omega_{3}+\omega_{4}$ in the case of longitudinal phase-matching. Curves $L$, $\omega_{1}=\omega_{L}\left(\lambda_{L}=1054 \mathrm{~nm}\right)$; curves SH, $\omega_{1}=\omega_{S H} \quad\left(\lambda_{S H}=527 \mathrm{~nm}\right)$. Dashdotted line indicates maximum allowed transversal wavevector mismatch for pump laser beam diameter of $d=5 \mu \mathrm{m}$ (self-focused or selftrapped beam).

frequency $\omega_{m, v}$. It is related to the peak Raman gain factor $g_{R, m}$ by [54]

$$
\chi_{R, m}^{\prime \prime}=\frac{1}{6} \frac{n_{1} n_{R} \epsilon_{0} c_{0}^{2}}{2 \omega_{R, m}} g_{R, m}
$$

where $\omega_{R, m}=\omega_{1}-\omega_{v, m}$. The polarized spontaneous Raman spectrum of $\mathrm{D}_{2} \mathrm{O}$ is shown in Fig. 17a (redrawn from [87]). It resembles the spectral distribution of the Raman scattering susceptibility. The main Raman band in the vibrational frequency region around $2450 \mathrm{~cm}^{-1}$ is composed of several vibrational modes $[68,71,87]$ whereby the symmetric Fermi resonance mode at $\tilde{\nu}_{v, 1}=2 \nu_{2 s}^{\prime}=2380 \mathrm{~cm}^{-1}$ and the symmetric O-D stretching mode at $\tilde{\nu}_{v, 2}=\nu_{s s}^{\prime}=2486 \mathrm{~cm}^{-1}$ dominate. Here we consider only the main Raman band and approximate it by two transitions of equal peak susceptibility $\chi_{R}^{\prime \prime}$ at the spectral positions $\tilde{\nu}_{R}=\tilde{\nu}_{1}-2380 \mathrm{~cm}^{-1}$ and $\tilde{\nu}_{R^{\prime}}=\tilde{\nu}_{1}-2486 \mathrm{~cm}^{-1}$. The spectral width of each of the two modes is set to $2 \Gamma / 2 \pi c_{0}=160 \mathrm{~cm}^{-1}$. A peak Raman gain factor of $\mathrm{H}_{2} \mathrm{O}$ was reported to be $g_{R}=7 \times 10^{-13} \mathrm{~m} \mathrm{~W}^{-1}$ for $\lambda_{1}=694.3 \mathrm{~nm}[3,88]$, giving $\chi_{k}^{\prime \prime}-3.9 \times 10^{23} \mathrm{~m}^{2} \mathrm{~V}^{2}$ by use of Equation $10\left(\tilde{\nu}_{1}\left(\mathrm{H}_{2} \mathrm{O}\right)=3260 \mathrm{~cm}^{-1}\right.$ is used [3]). Here we assume the same value of $\backslash_{R}^{\prime \prime}$ for $\mathrm{D}_{2} \mathrm{O}$. The discussed nonlinear optical parameters of $\mathrm{D}_{2} \mathrm{O}$ are collected in Table I.

The parametric nonlinear susceptibilities $\chi^{(3)}\left(-\omega_{3} ; \omega_{1}, \omega_{1},-\omega_{4}\right)$ and $\chi^{(3)}\left(-\omega_{4} ; \omega_{1}\right.$, 


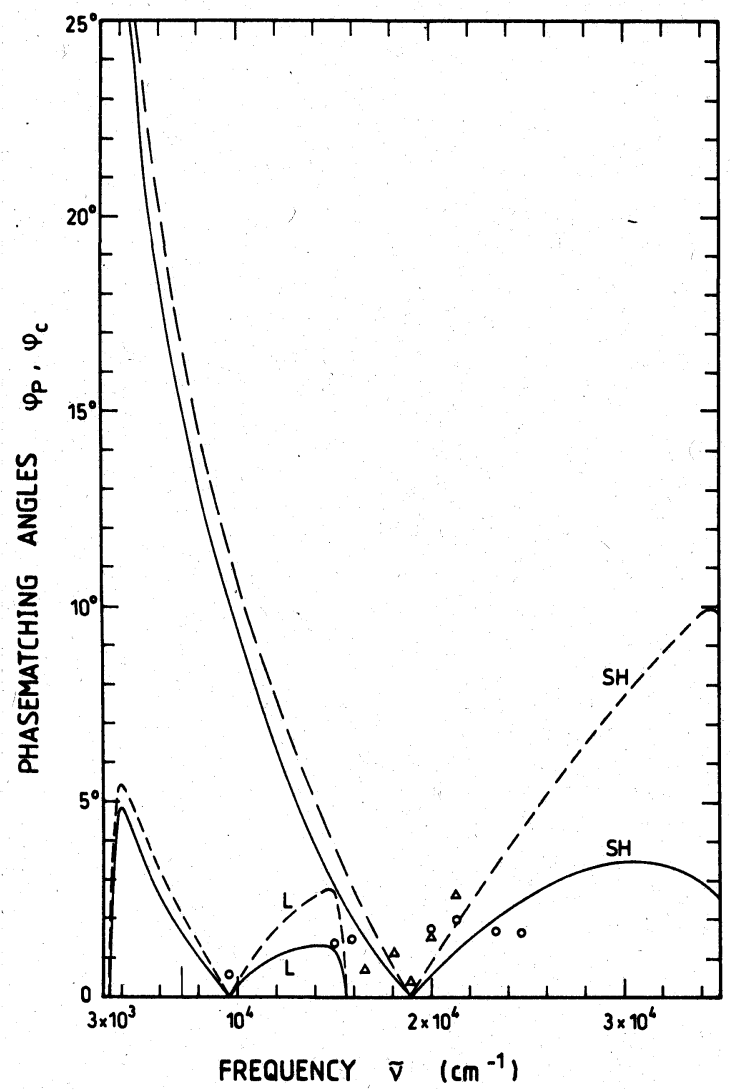

Figure 15 Internal noncollinear phase-matching angles $\varphi_{P}$ (solid curves) and maximum longitudinal phase-matching angles $\varphi_{c}$ (dashed curves) for interaction $\omega_{1}+\omega_{1} \rightarrow \omega_{3}+\omega_{4}$. L, $\omega_{1}=\omega_{L}\left(\lambda_{L}=1054 \mathrm{~nm}\right) . S H, \omega_{1}=\omega_{S H}\left(\lambda_{S H}=\right.$ $527 \mathrm{~nm})$. The experimentally.determined external divergence angles are included (half-widths at half-maximum outside the sample). Circles: $\lambda_{L}=1054 \mathrm{~nm}$. Triangles: $\lambda_{S H}=527 \mathrm{~nm}$.

$\left.\omega_{1},-\omega_{3}\right)$ are approximated by [23]

$$
\begin{aligned}
\chi^{(3)}\left(-\omega_{3} ; \omega_{1}, \omega_{1},-\omega_{4}\right)= & \chi^{(3)^{*}}\left(-\omega_{4} ; \omega_{1}, \omega_{1},-\omega_{3}\right) \\
& \approx \chi_{N R}^{(3)}+\chi_{R}^{(3)}\left(-\omega_{3} ; \omega_{1}, \omega_{1},-\omega_{4}\right) \\
& \approx \chi_{N R}^{(3)}+\frac{\chi_{R}^{\prime \prime}}{f} \Gamma\left[\frac{\left(\omega_{v 1}-\omega_{1}+\omega_{4}\right)-\mathrm{i} \Gamma}{\left(\omega_{v 1}-\omega_{1}+\omega_{4}\right)^{2}+\Gamma^{2}}+\frac{\left(\omega_{v 2}-\omega_{1}+\omega_{4}\right)-\mathrm{i} \Gamma}{\left(\omega_{v 2}-\omega_{1}+\omega_{4}\right)^{2}+\Gamma^{2}}\right]
\end{aligned}
$$

where $\chi_{N R}^{(3)}$ is the third-order nonresonant susceptibility and $f=1+\Gamma^{2} /\left[\left(\omega_{v 2}-\omega_{v 1}\right)^{2}+\Gamma^{2}\right]$ is a normalizing factor. The nonresonant susceptibility of $\mathrm{H}_{2} \mathrm{O}$ was determined previously by third-harmonic generation [89]. A value of $\chi_{N R}^{(3)}=2.2 \times 10^{-23} \mathrm{~m}^{2} \mathrm{~V}^{-2}$ was obtained. The same value is assumed here for $\mathrm{D}_{2} \mathrm{O}$. In Fig. 17b the approximate dispersion of $i^{(3)}\left(-\omega_{3}: \omega_{1}, \omega_{1},-\omega_{4}\right)=\gamma^{(3)^{\prime}}\left(-\omega_{3} ; \omega_{1}, \omega_{1},-\omega_{4}\right)-\mathrm{i} \chi_{R}^{(3)^{\prime \prime}}\left(-\omega_{3} ; \omega_{1}, \omega_{1},-\omega_{4}\right)$ is shown (curves are valid for both $\lambda_{1}=1054 \mathrm{~nm}$ and $\lambda_{1}=\lambda_{S H}=527 \mathrm{~nm}$ ). The values $\chi_{R}^{\prime \prime}=3.9 \times$ 10 ${ }^{23} \mathrm{~m}^{2} \mathrm{~V}^{2}$ and $\chi_{N R}^{(3)}=2.2 \times 10^{-2.3} \mathrm{~m}^{2} \mathrm{~V}^{-2}$ give a ratio of $b=\chi_{R}^{\prime \prime} / \chi_{N R}^{(3)} \approx 1.8$ : In [90] the nonlinear susceptibility ratio $b$ of $\mathrm{H}_{2} \mathrm{O}$ was determined by CARS spectroscopy and a value of $b=2$ was obtained, in good agreement with the ratio used here.

The following Sections 4.2 to 4.5 discuss separately the various processes covered by the equation system 13 and give order-of-magnitude estimates of their contributions. 


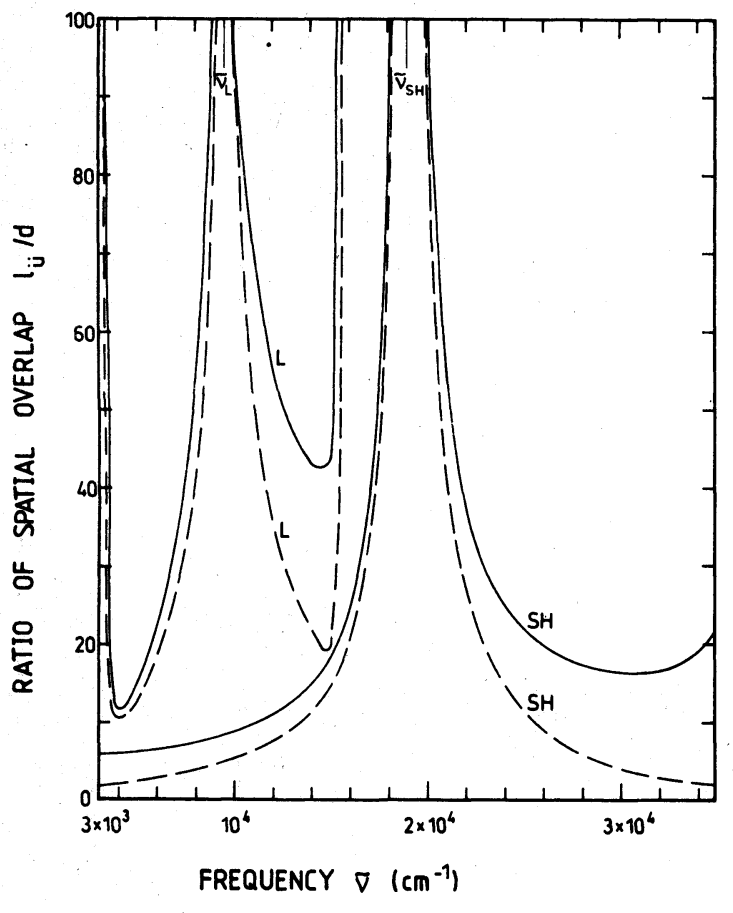

Figure 16 Ratio of spatial overlap of pump light and generated light in the interaction process $\omega_{1}+\omega_{1} \rightarrow \omega_{3}+\omega_{4}$. Solid curves, non-collinear phase-matched interaction. Dashed curves, longitudinally phasematched interactions with maximum transversal phase-mismatch $\left(\varphi_{3}=\varphi_{3 c}, \varphi_{4}=0\right.$ and vice versa).

\subsection{Stimulated Raman scattering}

The stimulated Raman scattering process $\omega_{1} \rightarrow \omega_{R}+\omega_{v}$ is described by

$$
\frac{\partial E_{R}}{\partial z}=\frac{\omega_{S}}{2 n_{R} c_{0}} 6 \chi_{R}^{\prime \prime}\left|E_{1}\right|^{2} E_{R}
$$

Absorptive losses and pump pulse depletion are neglected in Equation $12\left(\alpha_{1}=0\right.$, $\alpha_{R}=0, E_{1}=E_{1}(0)=$ const.). The solution of Equation 12 gives [54]

$$
I_{R}=I_{R N}\left[\exp \left(g_{R} I_{1} l_{R}\right)-1\right]
$$

where $I_{R}=\left(n_{R} \epsilon_{0} c_{0} / 2\right)\left|E_{R}\right|^{2}$ is the generated Raman intensity, $I_{1}=\left(n_{1} \epsilon_{0} c_{0} / 2\right)\left|E_{1}\right|^{2}$ is the pump laser intensity $\left(I\right.$ in W ${ }^{-2}, E$ in $\left.\mathrm{V} \mathrm{m}^{-1}\right), l_{R}$ is the interaction length determined by the sample length $l$ or the filament length, and $g_{R}$ is the Raman gain factor (see Equation 10). $I_{R N}$ is the equivalent noise input. It is given by [54]

$$
I_{R N} \approx \frac{\hbar \omega_{R}^{3} n_{R}^{3}}{(2 \pi)^{3} c_{0}^{2}} \Delta \Omega \Delta \omega_{R}=h \tilde{\nu}_{R}^{3} n_{R}^{3} c_{0}^{2} \pi \theta_{1 / 2}^{2} \Delta \tilde{\nu}_{R}
$$

where $\Delta \Omega=\pi \theta_{1 / 2}^{2}$ is the solid angle of efficient stimulated Raman scattering; $\theta_{1 / 2}$ is the divergence angle of the stimulated Stokes Raman emission (HWHM); $h$ is the Planck constant and $\hbar=h / 2 \pi ; I_{R N} \approx 0.3 \mathrm{~W} \mathrm{~cm}^{-2}$ is calculated for $\tilde{\nu}_{R}=\tilde{\nu}_{S H}-\tilde{\nu}_{v}=16595 \mathrm{~cm}^{-1}$, $\Delta \tilde{\nu}_{R}=160 \mathrm{~cm}^{-1}$, and $\theta_{1 / 2}=10^{-2} \mathrm{rad}$. The threshold pump intensity for efficient stimu- 

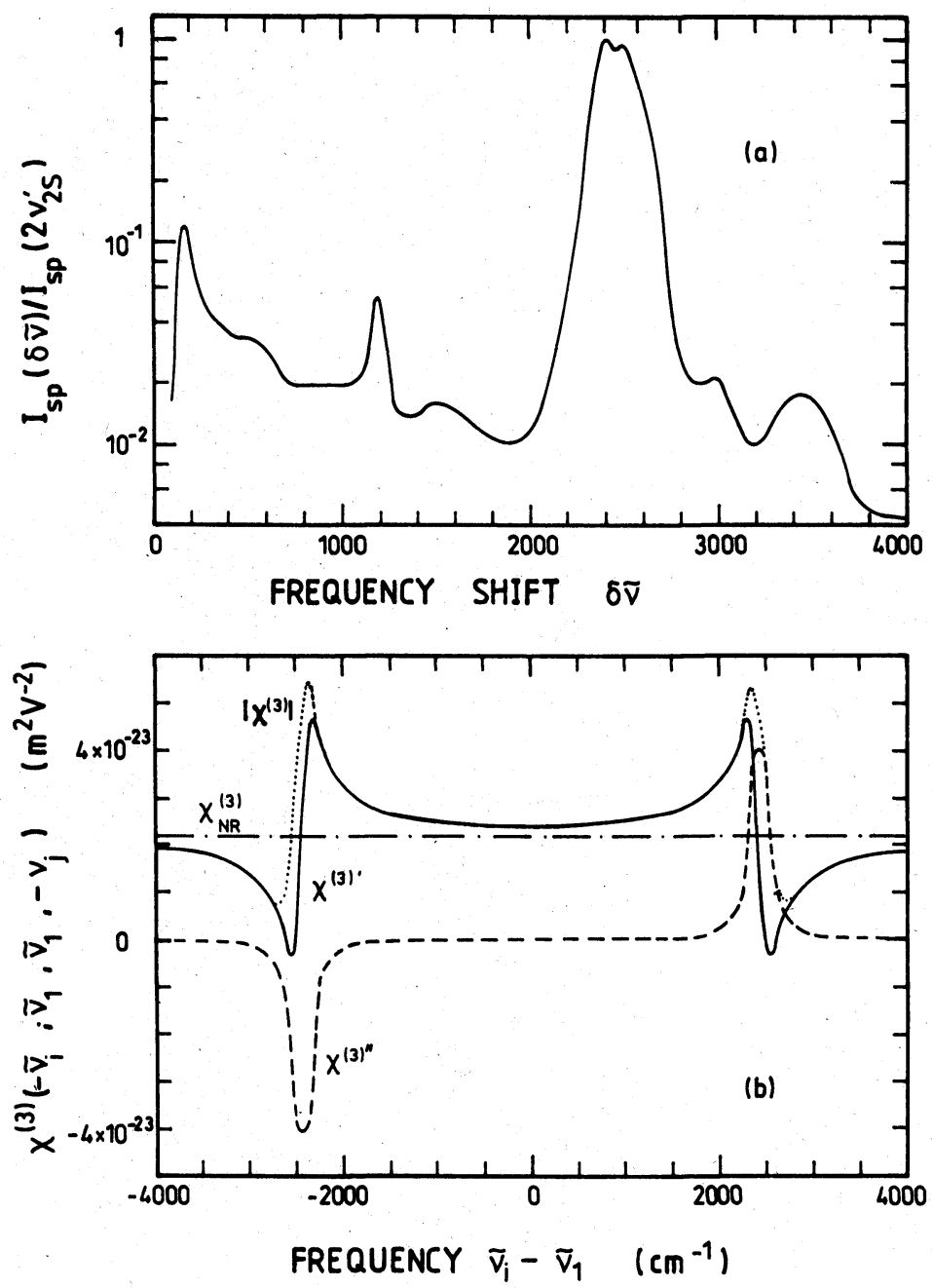

Figure 17 (a) Polarized spontaneous Raman spectrum of $\mathrm{D}_{2} \mathrm{O}$ (redrawn from [87]). (b) Approximate dispersion of third-order nonlinear susceptibility $\chi^{(3)}\left(-\tilde{\nu}_{i}, \tilde{\nu}_{1} ; \tilde{\nu}_{1},-\tilde{\nu}_{j}\right): i=3$ and $j=4$ for $\tilde{\nu}_{i}-\tilde{\nu}_{1}>0$; $i=4$ and $j=3$ for $\tilde{\nu}_{i}-\tilde{\nu}_{1}<0$. Solid curve, real part $\chi^{(3)^{\prime}}$. Dashed curve, imaginary part $\chi^{(3)^{\prime \prime}}$. Dotted curve, absolute value $\left|\chi^{(3)}\right|$. Dash-dotted line, nonresonant contribution $\chi_{N R}^{(3)}$.

lated Raman scattering is

$$
I_{1, \mathrm{th}}^{(R)}=\frac{\ln \left(I_{R} / I_{R N}\right)}{g_{R} l_{R}}=\frac{\ln \left(0.01 I_{1, \mathrm{~h}}^{(R)} / I_{R N}\right)}{g_{R} l_{R}} \approx \frac{20}{g_{R} l_{R}}
$$

where $I_{R}=0.01 I_{1, \mathrm{th}}^{(R)}$ has been set. A value of $I_{1, \mathrm{th}}^{(R)} \approx 6 \times 10^{10} \mathrm{~W} \mathrm{~cm}^{-2}$ is obtained for $g_{R}=7 \times 10^{-11} \mathrm{~cm} \mathrm{~W}^{-1}$ and $l_{R}=l=5 \mathrm{~cm}$. A threshold pump light peak intensity of $I_{S H, \text { th }}^{(R)} \approx 1.4 \times 10^{10} \mathrm{~W} \mathrm{~cm}^{-2}$ was determined in our experiments with second-harmonic pump light and an $80-\mathrm{cm}$ input focusing lens. The low experimental threshold intensity 
TA B LE I Nonlinear optical parameters of $\mathrm{D}_{2} \mathrm{O}$ applied in calculations

\begin{tabular}{lll}
\hline Parameter & Value & Ref. \\
\hline$\chi_{\mathrm{nl}}^{(3)^{\prime}}$ & $9.8 \times 10^{-23} \mathrm{~m}^{2} \mathrm{~V}^{-2}$ & Eq. 7 [22] \\
$n_{2, \mathrm{nl}}(527 \mathrm{~nm})$ & $2.2 \times 10^{-22} \mathrm{~m}^{2} \mathrm{~V}^{-2}$ & Eq. 7 [22] \\
$g_{R}\left(\lambda_{L}=694.3 \mathrm{~nm}\right)$ & $7 \times 10^{-13} \mathrm{~m} \mathrm{~W}^{-1}$ & {$[3]^{\mathrm{a}}$} \\
$\chi_{R}^{\prime \prime}$ & $3.9 \times 10^{-23} \mathrm{~m}^{2} \mathrm{~V}^{-2}$ & Eq. $10^{\mathrm{a}}$ \\
$\chi_{N R}^{(3)}$ & $2.2 \times 10^{-23} \mathrm{~m}^{2} \mathrm{~V}^{-2}$ & {$[89]^{\mathrm{a}}$} \\
\hline
\end{tabular}

a Value for $\mathrm{H}_{2} \mathrm{O}$. It is assumed to be the same for $\mathrm{D}_{2} \mathrm{O}$.

compared to the theoretical threshold intensity indicates the occurrence of self-focusing in our experiments.

\subsection{Pump-pulse-degenerate parametric four-photon interaction}

The pump-pulse-degenerate parametric four-photon interaction neglecting pump pulse depletion, absorption losses, and nonlinear refractive index contributions is described by [23]

$$
\begin{aligned}
& \frac{\partial E\left(\omega_{3}\right)}{\partial z}=-\mathrm{i}\left(\omega_{3} / 2 n_{3} c_{0}\right) 3 \chi^{(3)}\left(-\omega_{3} ; \omega_{1}, \omega_{1},-\omega_{4}\right) E_{1}^{2} E^{*}\left(\omega_{4}\right) \exp (\mathrm{i} \Delta \mathbf{k r}) \\
& \frac{\partial E\left(\omega_{4}\right)}{\partial z}=-\mathrm{i}\left(\omega_{4} / 2 n_{4} c_{0}\right) 3 \chi^{(3)^{*}}\left(-\omega_{3} ; \omega_{1}, \omega_{1},-\omega_{4}\right) E_{1}^{2} E^{*}\left(\omega_{3}\right) \exp (\mathrm{i} \Delta \mathbf{k r})
\end{aligned}
$$

$E\left(\omega_{3}\right)$ and $E\left(\omega_{4}\right)$ are the electric field strengths per frequency interval (units $\mathrm{V} \mathrm{m}^{-1} \mathrm{~s}$, for example) of the signal and the idler light, respectively. In the case of collinear nonphase-matched interaction $(\Delta \mathbf{k r}=\Delta k z)$ without signal and idler input, the solution of Equations (16) and (17) is [23]

$$
\begin{aligned}
& I\left(\omega_{3}, l\right)=I_{N}\left(\omega_{4}\right)\left(\left|\kappa_{3}\right|^{2} /|\beta|^{2}\right)\left|\sinh \left(\beta l_{P}\right)\right|^{2} \\
& I\left(\omega_{4}, l\right)=I_{N}\left(\omega_{3}\right)\left(\left|\kappa_{4}\right|^{2} /|\beta|^{2}\right)\left|\sinh \left(\beta l_{P}\right)\right|^{2}
\end{aligned}
$$

with

$$
\begin{aligned}
\kappa_{3} & =\left(\omega_{3} / 2 n_{3} c_{0}\right) 3 \chi^{(3)}\left(-\omega_{3} ; \omega_{1}, \omega_{1},-\omega_{4}\right) E_{1}^{2} \\
\kappa_{4} & =\left(\omega_{4} / 2 n_{4} c_{0}\right) 3 \chi^{(3)^{*}}\left(-\omega_{3} ; \omega_{1}, \omega_{1},-\omega_{4}\right) E_{1}^{2} \\
\beta & =\left(\kappa_{3}^{*} \kappa_{4}-\frac{1}{4} \Delta k^{2}\right)^{1 / 2}
\end{aligned}
$$

$l_{P} \leq l$ is the length of high pump laser intensity (filament length in the case of self-focusing). $I\left(\omega_{3}\right)$ and $I\left(\omega_{4}\right)$ are the spectral energy densities (units $\mathrm{J} \mathrm{m}^{-2} \mathrm{~s}$, for example) of the signal and idler light, respectively. $I\left(\omega_{i}\right)$ is related to $E\left(\omega_{i}\right)$ by [54] $I\left(\omega_{i}\right)=\left(n_{i} \epsilon_{0} c_{0} / \pi\right)\left|E\left(\omega_{i}\right)\right|^{2}$. $I_{N}\left(\omega_{4}\right)$ and $I_{N}\left(\omega_{3}\right)$ represent the quantum fluctuations at the idler frequency $\omega_{4}$ and the signal frequency $\omega_{3}$, respectively. $I_{N}\left(\omega_{i}\right)=(2 \pi)^{-1} I_{N}\left(\nu_{i}\right)=\left(2 \pi c_{0}\right)^{-1} I_{N}\left(\tilde{\nu}_{i}\right)$ is given approximately by $[23,54]$

$$
I_{N}\left(\omega_{i}\right) \approx\left[\hbar \omega_{i}^{3} n_{i}^{3} /(2 \pi)^{3} c_{0}^{2}\right] \Delta \Omega \Delta t_{1}
$$

or

$$
I_{N}\left(\tilde{\nu}_{i}\right) \approx h \tilde{\nu}_{i}^{3} c_{0}^{2} n_{i}^{3} \pi \theta_{1 / 2}^{2} \Delta t_{1}
$$


As an example we estimate $I_{N}\left(\tilde{\nu}_{4}\right) \approx 7 \times 10^{-14} \mathrm{~J} \mathrm{~cm}^{-1}$ for $\tilde{\nu}_{4}=17000 \mathrm{~cm}^{-1}, \theta_{1 / 2}=0.025 \mathrm{rad}$ and $\Delta t_{1}=5 \mathrm{ps}$. At $I_{1}=I_{1, \text { th }}^{(R)}=6 \times 10^{10} \mathrm{~W} \mathrm{~cm}^{-2}\left(w_{1, \text { th }}^{(R)}=I_{1, \text { th }}^{(R)} \Delta t_{1}=0.3 \mathrm{~J} \mathrm{~cm}^{-2}\right)$ it is $I_{N}\left(\tilde{\nu}_{4}\right) / w_{1, \text { th }}^{(R)} \approx 2 \times 10^{-13} \mathrm{~cm}$ and $I_{N}\left(\tilde{\nu}_{4}\right) \Delta \tilde{\nu} / w_{1, \text { th }}^{(R)} \approx 10^{-9}$ for a light continuum extension of $\Delta \tilde{\nu} \approx 5000 \mathrm{~cm}^{-1}$. For a conversion efficiency of $I(\tilde{\nu}) \Delta \tilde{\nu} / w_{1}=0.01$, a gain of $I(\tilde{\nu}) / I_{N}(\tilde{\nu}) \approx\left|\sinh \left(\beta l_{p}\right)\right|^{2} \approx \exp \left[\left(\beta+\beta^{*}\right) l_{P}\right]=\exp \left[2 \operatorname{Re}(\beta) l_{P}\right] \approx 10^{7}$, or a gain coefficient $G=2 \operatorname{Re}(\beta) l_{P} \approx 16$ is necessary. In the case of $\chi^{(3)}$ real $\left(\kappa_{3}, \kappa_{4}\right.$ real), $\beta$ is pure imaginary and no amplification occurs if $\kappa_{3} \kappa_{4}<\Delta k^{2} / 4$. When $\chi^{(3)}$ is pure imaginary $\left(\chi^{(3)^{\prime}}=0\right)$ it is $\kappa_{3}^{*} \kappa_{4}$ negative and $\beta$ is pure imaginary (no amplification). In Fig. 18 the gain coefficient $G$ is plotted versus frequency for three pump pulse intensities. The curves apply to $\nu_{1}=\nu_{S H}, l_{P}=l=5 \mathrm{~cm}$ and the third-order nonlinear susceptibility of Fig. $17 \mathrm{~b}$. In the frequency region around the pump laser frequency the wavevector mismatch $\Delta k$ is small (Fig. 14) and the gain coefficient is high. For $\Delta k=0$ a threshold intensity of $I_{S H, \text { th }}^{(P)} \approx 8 \times 10^{10} \mathrm{~W} \mathrm{~cm}^{-2}$ is estimated for efficient parametric four-photon interaction. With rising pump pulse intensity the frequency interval of high gain broadens (effect of phase-mismatch reduces). The resonant structure of $\chi^{(3)}$ shows up in the frequency dependence of $G$ around $\tilde{\nu}_{R}$ and $\tilde{\nu}_{A}$.

In the case of noncollinear phase-matched interaction $(\Delta \mathbf{k r}=0)$, Equations 18 and 19 reduce to

with

$$
\begin{aligned}
& I\left(\omega_{3}, l\right) \approx I_{N}\left(\omega_{4}\right)\left(\omega_{3} n_{4} / \omega_{4} n_{3}\right)\left|\sinh \left(\kappa l_{\ddot{u}, 4, P}\right)\right|^{2}\left(l_{P} / l_{\ddot{u}, 4, P}\right) \\
& I\left(\omega_{4}, l\right) \approx I_{N}\left(\omega_{3}\right)\left(\omega_{4} n_{3} / \omega_{3} n_{4}\right)\left|\sinh \left(k l_{\ddot{u}, 3, P}\right)\right|^{2}\left(l_{P} / l_{\ddot{u}, 3, P}\right)
\end{aligned}
$$

$$
\begin{aligned}
\kappa & =\beta(\Delta k=0)=\left(\kappa_{3}^{*} \kappa_{4}\right)^{1 / 2}=\left[\omega_{3} \omega_{4} / n_{3} n_{4}\right]^{1 / 2}\left(3 / 2 c_{0}\right) \chi^{(3)^{*}}\left(-\omega_{3} ; \omega_{1}, \omega_{1},-\omega_{4}\right)\left|E_{1}\right|^{2} \\
& =\left[\omega_{3} \omega_{4} / n_{3} n_{4}\right]^{1 / 2}\left(3 / n_{1} \epsilon_{0} c_{0}^{2}\right) \chi^{(3)^{*}}\left(-\omega_{3} ; \omega_{1}, \omega_{1},-\omega_{4}\right) I_{1}
\end{aligned}
$$

The overlap lengths $l_{i \vec{u}, 3, P}$ and $l_{i \dot{u}, 4, P}$ are shown by the solid curves in Fig. 16. After a length interval of $l_{\ddot{u}, i, P}$ the generated signal and idler light runs out of the overlap region with the pump light. The factors $l_{P} / l_{\ddot{u}, i, P}$ take care of the fact that the parametric interaction restarts after running out of overlap and the output signal and idler light add up. Efficient light continuum generation $\left[I(\tilde{\nu}) \Delta \tilde{\nu} / w_{1} \approx 0.01\right]$ requires $I\left(\omega_{3}, l\right) / I_{N}\left(\omega_{4}\right) \approx 10^{7}$. Insertion into Equation 24 gives $\left.\left|\sinh \left(\kappa l_{\ddot{u}, 4, P}\right)\right|^{2} \approx \exp \left[\left(\kappa+\kappa^{*}\right) l_{\ddot{u}, 4, P}\right)\right] \approx 10^{7} l_{\ddot{u}, 4, P} / l_{P}$ or $\operatorname{Re}(\kappa)=[8+$ $\left.\ln \left(l_{i, 4, P} / l_{P}\right)\right] / l_{i, 4, P}$. For $\tilde{\nu}_{3}=\tilde{\nu}_{S H}+1000 \mathrm{~cm}^{-1}$ it is $l_{\dot{u}, 4, P} \approx 0.4 \mathrm{~mm}(d=5 \mu \mathrm{m}$, Fig. 16) leading to $\operatorname{Re}(\kappa) \approx 80 \mathrm{~cm}^{-1}\left(l_{P}=l=5 \mathrm{~cm}\right)$ and $I_{S H, \text { th }}^{(P)} \approx 4 \times 10^{12} \mathrm{~W} \mathrm{~cm}^{-2}\left(\chi^{(3)}=\chi^{(3)^{\prime}}=\right.$ $2.5 \times 10^{-23} \mathrm{~V} \mathrm{~m}^{-2}$, see Fig. $\left.17 \mathrm{~b}\right)$. The high threshold intensity makes noncollinear phasematched parametric four-photon interaction under self-focusing conditions (small beam diameter $d$, short interaction length) unlikely.

For the longitudinally phase-matched Cerenkov-like parametric four-photon interaction in filaments $[\exp (i \Delta \mathbf{k r}) \approx 1$ ], the interaction is enhanced because of phase-matching, but the interaction length $l_{i}$ is shortened by noncollinear signal-idler propagation. For the case of $l_{i \ddot{i}, 4}<l_{i, 3}$ the parametric generation is described by (see Appendix)

$$
\begin{aligned}
& I\left(\omega_{3}, l\right) \approx I_{N}\left(\omega_{4}\right) \frac{\omega_{3}}{\omega_{4}}\left|\sinh \left(\kappa l_{\ddot{u}, 4}\right)\right|^{2}\left|\frac{\left[\cosh \left(\kappa l_{\ddot{u}, 4}\right)\right]_{l_{u, 3} / l_{u, 4}}-1}{\cosh \left(\kappa l_{\ddot{u}, 4}\right)-1}\right|^{2} \frac{l_{P}}{l_{\ddot{u}, 3}} \\
& I\left(\omega_{4}, l\right) \approx I_{N}\left(\omega_{4}\right)\left|\sinh \left(\kappa l_{\ddot{u}, 4}\right)\right|^{4}\left|\frac{\cosh \left(\kappa l_{\ddot{u}, 4}\right)}{\cosh \left(\kappa l_{\ddot{u}, 4}\right)-1}\right|^{2} \frac{\left|\cosh \left(\kappa l_{i, 4}\right)\right|^{2\left(l_{u, 3} / l_{u, 4}-1\right)}-1}{\left|\cosh \left(\kappa l_{\ddot{u}, 4}\right)\right|^{2}-1} \frac{l_{P}}{l_{\ddot{u}, 3}}
\end{aligned}
$$


Only the most important term of Equation A11 at high gain is retained in Equation 28. For the case of $l_{i u, 4}>l_{\ddot{u}, 3}$ the indices 3 and 4 in Equations 27 and 28 have to be interchanged. $\kappa$ is given by Equation 26 . The length $l_{P}$ is the filament length $\left(l_{P} \leq l\right)$. In the extreme cases of (i) $\varphi_{3}=0, \varphi_{4}=\varphi_{4, c}$ and (ii) $\varphi_{3}=\varphi_{3, c}, \varphi_{4}=0$ the overlap lengths are (i) $l_{\ddot{u}, 3}=l_{P}, l_{\ddot{u}, 4}=l_{\ddot{u}, 4, c}$ and (ii) $l_{\ddot{u}, 3}=l_{\ddot{u}, 3, c}, l_{\ddot{u}, 4}=l_{P}$.

For efficient longitudinally phase-matched parametric four-photon interaction $\operatorname{Re}(\kappa) l_{i, i}$ $(i=3,4)$ of the order of 0.3 is necessary. At $I_{1}=5 \times 10^{11} \mathrm{~W} \mathrm{~cm}^{-2}, \tilde{\nu}_{1}=$ $\lambda_{S H}^{-1}=18975 \mathrm{~cm}^{-1}, \tilde{\nu}_{3}=\tilde{\nu}_{1}+1500 \mathrm{~cm}^{-1}, \tilde{\nu}_{4}=\tilde{\nu}_{1}-1500 \mathrm{~cm}^{-1}, \varphi_{3}=0, \varphi_{4}=\varphi_{4 c}, l_{\ddot{u}, 4}=$ $0.29 \mathrm{~mm}$ (Fig. 16 with $d=5 \mu \mathrm{m}$ ), and $l_{P}=l=5 \mathrm{~cm}$ we estimate $\kappa l_{i, 3} \approx$ $0.295\left[\chi^{(3)}\left(-\omega_{3} ; \omega_{1}, \omega_{1},-\omega_{4}\right)=2.68 \times 10^{-23} \mathrm{~m}^{2} \mathrm{~V}^{-2}\right], \quad I\left(\tilde{\nu}_{3}, l\right) / I_{N}\left(\tilde{\nu}_{4}\right) \approx 1.5 \times 10^{8} \quad$ and $I\left(\tilde{\nu}_{4}, l\right) / I_{N}\left(\tilde{\nu}_{4}\right) \approx 1.3 \times 10^{8}$. For the same pump pulse intensity of $I_{S H}=5 \times 10^{11} \mathrm{~W} \mathrm{~cm}^{-2}$ and the same frequency of $\tilde{\nu}_{3}=\tilde{\nu}_{S H}+1500 \mathrm{~cm}^{-1}$ the collinear non-phase matched parametric four-photon interaction gives $I\left(\omega_{3}, l\right) / I_{N}\left(\omega_{4}\right) \approx I\left(\omega_{4}, l\right) / I_{N}\left(\omega_{3}\right) \approx 10^{-2}$ (Equations 18 and 19 with $\left.\operatorname{Re}(\beta) l_{P}=0.111\right)$. This example shows the dominance of the longitudinally phase-matched Čerenkov-like interaction compared to the collinear non-phase-matched interaction at the selected pump pulse intensity and frequency.

In Fig. 19, $I\left(\tilde{\nu}_{i}, l, \varphi_{i}=0\right) \tilde{\nu}_{j} / I_{N}\left(\tilde{\nu}_{j}\right) \tilde{\nu}_{i}$ of Equation 27 is plotted versus frequency $\tilde{\nu}_{i}(i=3$ for $\tilde{\nu}_{i}>\tilde{\nu}_{S H}, i=4$ for $\tilde{\nu}_{i}<\tilde{\nu}_{S H}$ ) for three different pump laser intensities. The Raman resonant structure of $\chi^{(3)}$ is imprinted in the frequency-dependent amplification behaviour. A comparison of Figs 18 and 19 shows that outside a central frequency region around the pump laser frequency the longitudinally phase-matched interaction is generally more efficient than the collinear non-phase-matched interaction. The spectrum displayed in Fig. 10a strongly resembles the gain profiles of Fig. 19, especially of the curves 2 and 3.

Since $I_{1}^{(R)}$ th $\left(l_{R}=5 \mathrm{~cm}\right)$ is only $6 \times 10^{10} \mathrm{~W} \mathrm{~cm}^{-2}$, the stimulated Raman scattering dominates for long interaction lengths. For the Čerenkov-like longitudinally phase-matched parametric four-photon interaction with a fixed product of $I_{1} l_{P}$ it is favourable to have high pump intensities $I_{1}$ (in order to achieve $\kappa l_{\ddot{u}} \approx 0.3$ ) and short interaction length $l_{P}$ (under the condition of a short interaction length $l_{R}$ the Raman threshold intensity rises).

The threshold intensity $I_{1, \text { th }}^{(P)}$ of efficient Čerenkov-like, longitudinally phase-matched . pump-pulse-degenerate parametric four-photon interaction is of the order of $5 \times 10^{11} \mathrm{~W} \mathrm{~cm}^{-2}$ (see Fig. 19). This value should be compared with the critical intensity of optical dielectric breakdown $I_{B}$ in $\mathrm{D}_{2} \mathrm{O}$. In [22] a breakdown threshold intensity of $I_{B}\left(\Delta t=30 \mathrm{ps}, \lambda_{L}=1064 \mathrm{~nm}\right) \approx 8.2 \times 10^{11} \mathrm{~W} \mathrm{~cm}^{-2}$ was determined. $I_{B}\left(\lambda_{S H}=532 \mathrm{~nm}\right)$ was found to be somewhat larger than $I_{B}\left(\lambda_{L}=1064 \mathrm{~nm}\right)$ [22]. Assuming $I_{B}$ to be inversely proportional to the pulse duration for picosecond pulses [64], we estimate $I_{B}\left(\mathrm{D}_{2} \mathrm{O}, \Delta t=5 \mathrm{ps}\right) \approx 5 \times 10^{12} \mathrm{~W} \mathrm{~cm}^{-2}$. This is an order of magnitude larger than the estimated $I_{1, \text { th }}^{(P)}$.

\subsection{Coherent antiStokes Raman scattering}

The parametric Stokes--antiStokes coupling around the difference frequency resonance $\left|\omega_{1}-\omega_{4}\right|=\left|\omega_{3}-\omega_{1}\right| \approx \omega_{v}$ is already included in the parametric four-photon interaction (Section 4.3). If Stokes light is present that is not generated by the parametric four-photon interaction $\omega_{1}+\omega_{1} \rightarrow \omega_{3}+\omega_{4}$ (e.g. light generated by stimulated Raman scattering), it may be converted to the antiStokes side by coherent antiStokes Raman scattering (CARS) $\omega_{1}+\omega_{1}-\omega_{4} \rightarrow \omega_{3}[28,69,90]$. 


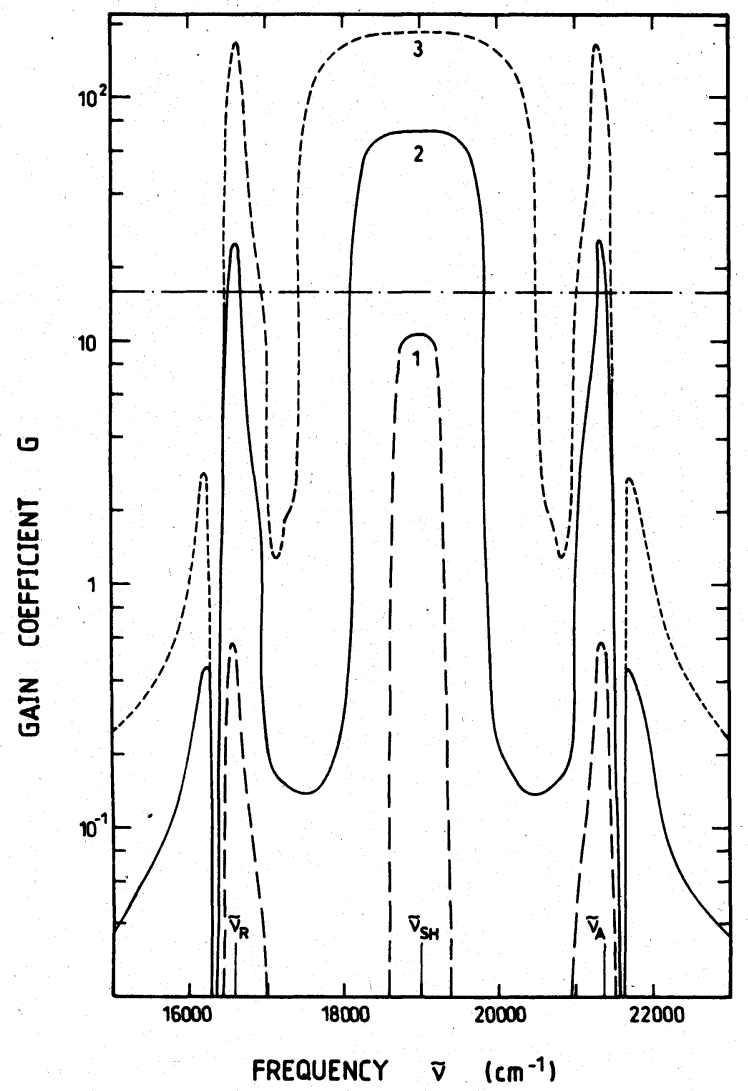

Figure 18 Gain coefficient $G=2 \operatorname{Re}(\beta) /$ of collinear pump-pulse-degenerate parametric interaction process $\tilde{\nu}_{S H}+\tilde{\nu}_{S H} \rightarrow \tilde{\nu}_{3}+\tilde{\nu}_{4}$ versus frequency $\tilde{\nu}\left(\tilde{\nu}\right.$ represents $\tilde{\nu}_{3}$ for $\tilde{\nu} \geq \tilde{\nu}_{S H}$ and $\tilde{\nu}_{4}$ for $\left.\tilde{\nu} \leq \tilde{\nu}_{S H}\right)$. The curves correspond to pump pulse intensities (1) $I_{S H}=6 \times$ $10^{10} \mathrm{~W} \mathrm{~cm}^{-2}$, (2) $4 \times 10^{11} \mathrm{~W} \mathrm{~cm}^{-2}$, and (3) $1 \times 10^{12} \mathrm{~W} \mathrm{~cm}^{-2}$. The dash-dotted line indicates the gain coefficient $G$ necessary for efficient parametric four-photon interaction (conversion efficiency $\approx 0.01$ ).

Neglecting pump pulse depletion and Stokes light depletion, the CARS process is described by

$$
\partial E\left(\omega_{3}\right) / \partial z=-\mathrm{i}\left(\omega_{3} / 2 n_{3} c_{0}\right) 3 \chi^{(3)}\left(-\omega_{3} ; \omega_{1}, \omega_{1},-\omega_{4}\right) E_{1}^{2} E^{*}\left(\omega_{4}\right) \exp (\mathrm{i} \Delta \mathbf{k r})
$$

Under longitudinally phase-matched Čerenkov-like conditions the generated antiStokes signal may be expressed by

$$
\begin{aligned}
I\left(\omega_{3}\right) & =\left(n_{3} \epsilon_{0} c_{0} / \pi\right)\left|E\left(\omega_{3}\right)\right|^{2} \\
& =\left(n_{3} c_{0} \epsilon_{0} / \pi\right)\left(\omega_{3}^{2} / 4 n_{3}^{2} c_{0}^{2}\right) 9\left|\chi^{(3)}\left(-\omega_{3} ; \omega_{1}, \omega_{1},-\omega_{4}\right)\right|^{2}\left|E_{1}\right|^{4}\left|E\left(\omega_{4}\right)\right|^{2} l_{\dot{u}, 3}^{2}\left(l_{P} / l_{i, 3}\right) \\
& =\left(\omega_{3}^{2} / n_{1}^{2} n_{3} n_{4} \epsilon_{0}^{2} c_{0}^{4}\right) 9\left|\chi^{(3)}\left(-\omega_{3} ; \omega_{1}, \omega_{1},-\omega_{4}\right)\right|^{2} I_{1}^{2} I\left(\omega_{4}\right) l_{u, 3} l_{P}
\end{aligned}
$$

$\left|\chi^{(3)}\left(-\tilde{\nu}_{3} ; \tilde{\nu}_{1}, \tilde{\nu}_{1},-\tilde{\nu}_{4}\right)\right|$ is displayed in Fig. 17b (dotted curve). The resonant structure of $\chi^{(3)}$ favours the CARS interaction at the long-wavelength side of the antiStokes frequency $\nu_{A}=\nu_{1}+\nu_{v}$. At $\tilde{\nu}_{3}=\tilde{\nu}_{1}+2200 \mathrm{~cm}^{-1}$ an amplification of $I\left(\omega_{3}, l\right) / I\left(\omega_{4}\right) \approx 0.40$ is expected for $I_{1, \mathrm{th}}^{R}=6 \times 10^{10} \mathrm{~W} \mathrm{~cm}^{-2}, l_{P}=l=5 \mathrm{~cm}$, and $l_{\ddot{u}, 3}=0.19 \mathrm{~mm}$ according to Fig. $16\left(\left|\mathrm{v}^{(3)}\right|=4 \times 10^{-23} \mathrm{~m}^{2} \mathrm{~V}^{-2}\right.$, Fig. 17b). At $\tilde{\nu}_{3}=\tilde{\nu}_{1}+2700 \mathrm{~cm}^{-1}$ the expected 
amplification is $I\left(\omega_{3}, l\right) / I\left(\omega_{4}\right) \approx 0.012$ for $I_{1, \text { th }}^{R}=6 \times 10^{10} \mathrm{~W} \mathrm{~cm}^{-2}, l_{P}=5 \mathrm{~cm}$, and $l_{i, 3}=0.16 \mathrm{~mm}\left(\left|\chi^{(3)}\right|=7.7 \times 10^{-24} \mathrm{~m}^{2} \mathrm{~V}^{-2}\right.$, Fig. 17b). At $\omega_{A}=2 \omega_{L}-\omega_{R}$ a strong antiStokes signal would be expected because the stimulated Stokes signal $I_{4}=I_{R}$ is maximal at $\omega_{R}$, but inverse Raman scattering hinders the build-up of a strong signal at $\omega_{A}$.

\subsection{Inverse Raman scattering}

The inverse Raman scattering process $\omega_{A} \rightarrow \omega_{L}+\omega_{v}$ is described by [54]

with the solution [54]

$$
\partial E_{A} / \partial z=-\left(\omega_{A} / 2 n_{A} c_{0}\right) 6 \chi_{R}^{\prime \prime}\left|E_{L}\right|^{2} E_{A}
$$

$$
I_{A}(l)=I_{A, 0} \exp \left[-\left(\omega_{A} / \omega_{S}\right) g_{R} I_{1} l_{A}\right]
$$

where $l_{A}$ is an effective length of high pump laser intensity after antiStokes light has been generated (e.g. by CARS interaction). The inverse Raman scattering is intrinsically phase-matched. AntiStokes Raman signal initially present is attenuated. For $I_{1}=I_{1, \text { th }}^{(R)}=$ $6 \times 10^{10} \mathrm{~W} \mathrm{~cm}^{-2}, l_{A}=1 \mathrm{~cm}$, and $g_{R}=7 \times 10^{-11} \mathrm{~cm} \mathrm{~W}^{-1}$ it is $I_{A} / I_{A, 0}=0.0044$ (calculated for $\lambda_{1}=\lambda_{S H}=527 \mathrm{~nm}$ ). The inverse Raman scattering process explains the absence of a strong antiStokes signal at $\omega_{A}=2 \omega_{1}-\omega_{R}=\omega_{1}+\omega_{v}$ despite the presence of a strong Stokes Raman signal at $\omega_{R}=\omega_{1}-\omega_{v}$.

\subsection{Pump-pulse-nondegenerate parametric four-photon interaction}

After stimulated Raman scattering has generated an intense light pulse at frequency $\omega_{R}$, the pump-pulse-nondegenerate parametric four-photon interaction $\omega_{1}+\omega_{R} \rightarrow \omega_{3^{\prime}}+\omega_{4^{\prime}}$ becomes active. For this process the collinear wavevector mismatch $\Delta k=k_{3^{\prime}}+k_{4^{\prime}}-$ $k_{1}-k_{R}$ in the region $\omega_{R}<\omega_{4^{\prime}}<\omega_{3^{\prime}}<\omega_{1}$ is negative and an intensity-dependent phasematching by contributions of the positive nonlinear refractive indices $n_{2, \text { nl }}\left(\omega_{i}, \omega_{i}\right)$ and $n_{2, \mathrm{nl}}\left(\omega_{i}, \omega_{j}\right)$ (Equation 7) becomes possible in this region $[51,58,63,77]$.

The collinear nondegenerate parametric four-photon interaction is described by the following equation system when absorption losses and pump pulse depletion at $\omega_{L}$ and $\omega_{R}$ are neglected:

$$
\begin{aligned}
\partial E\left(\omega_{3^{\prime}}\right) / \partial z= & -\left(\mathrm{i} \omega_{3^{\prime}} / 2 n_{3^{\prime}} c_{0}\right) 6\left[\chi^{(3)}\left(-\omega_{3^{\prime}} ; \omega_{1}, \omega_{R},-\omega_{4^{\prime}}\right) E_{1} E_{R} E^{*}\left(\omega_{4^{\prime}}\right) \exp (\mathrm{i} \Delta k z)\right. \\
& \left.+\chi^{(3)}\left(-\omega_{3^{\prime}} ; \omega_{1},-\omega_{1}, \omega_{3^{\prime}}\right)\left|E_{1}\right|^{2} E\left(\omega_{3^{\prime}}\right)+\chi^{(3)}\left(-\omega_{3^{\prime}}, \omega_{R},-\omega_{R}, \omega_{3^{\prime}}\right)\left|E_{R}\right|^{2} E\left(\omega_{3^{\prime}}\right)\right] \\
\partial E\left(\omega_{4^{\prime}}\right) / \partial z= & -\left(\mathrm{i} \omega_{4^{\prime}} / 2 n_{4^{\prime}} c_{0}\right) 6\left[\chi^{(3)^{*}}\left(-\omega_{3^{\prime}} ; \omega_{1}, \omega_{R},-\omega_{4^{\prime}}\right) E_{1} E_{R} E^{*}\left(\omega_{3^{\prime}}\right) \exp (\mathrm{i} \Delta k z)\right. \\
& \left.+\chi^{(3)}\left(-\omega_{4^{\prime}} ; \omega_{1},-\omega_{1}, \omega_{4^{\prime}}\right)\left|E_{1}\right|^{2} E\left(\omega_{4^{\prime}}\right)+\chi^{(3)}\left(-\omega_{4^{\prime}} ; \omega_{R},-\omega_{R}, \omega_{4^{\prime}}\right)\left|E_{R}\right|^{2} E\left(\omega_{4^{\prime}}\right)\right] .
\end{aligned}
$$

$$
\partial E_{1} / \partial z=-\mathrm{i}\left(\omega_{1} / 2 n_{1} c_{0}\right)\left[3 \chi^{(3)}\left(-\omega_{1} ; \omega_{1},-\omega_{1}, \omega_{1}\right)\left|E_{1}\right|^{2} E_{1}+6 \chi^{(3)}\left(-\omega_{1} ; \omega_{R},-\omega_{R}, \omega_{1}\right)\left|E_{R}\right|^{2} E_{1}\right]
$$

$$
\begin{aligned}
\partial E_{R} / \partial z= & -\mathrm{i}\left(\omega_{R} / 2 n_{R} c_{0}\right)\left[3 \chi^{(3)}\left(-\omega_{R} ; \omega_{R},-\omega_{R}, \omega_{R}\right)\left|E_{R}\right|^{2} E_{R}\right. \\
& \left.+6 \chi^{(3)}\left(-\omega_{R} ; \omega_{1},-\omega_{1}, \omega_{R}\right)\left|E_{1}\right|^{2} E_{R}\right]
\end{aligned}
$$




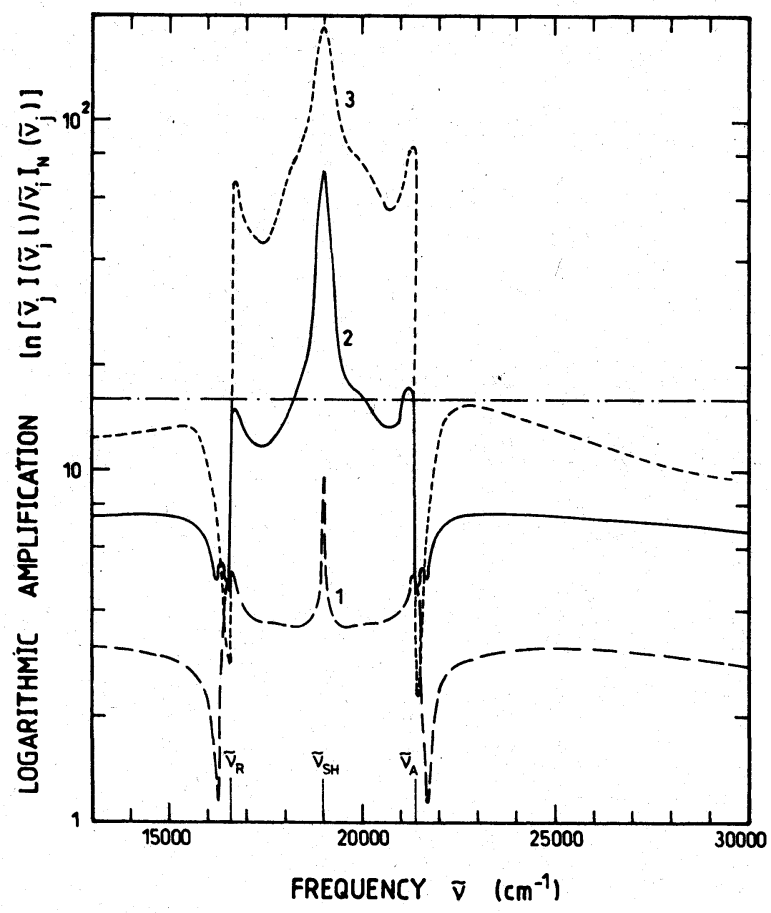

Figure 19 Plot of logarithmic gain $\ln \left[\tilde{\nu}_{j} /\left(\tilde{\nu}_{i}, I\right) / \tilde{\nu}_{i} l_{N}\left(\tilde{\nu}_{j}\right)\right]$ (Equation 27) for longitudinally phase-matched interaction $\tilde{\nu}_{S H}+$ $\tilde{\nu}_{S H} \rightarrow \tilde{\nu}_{3}+\tilde{\nu}_{4}$ versus $\tilde{\nu}_{i}$ for $\varphi_{i}=0\left(\tilde{\nu}_{i}=\tilde{\nu}_{3}\right.$ for $\tilde{\nu}_{i}>\tilde{\nu}_{S H}, \tilde{\nu}_{i}=\tilde{\nu}_{4}$ for $\tilde{\nu}_{i}<\tilde{\nu}_{S H}$ ). The curves correspond to pump pulse intensities of (1) $I_{S H}=6 \times 10^{10} \mathrm{~W} \mathrm{~cm}^{-2}$, (2) $4 \times 10^{11} \mathrm{~W} \mathrm{~cm}^{-2}$, (3) $1 \times 10^{12} \mathrm{~W} \mathrm{~cm}^{-2}$. The dash-dotted line indicates the logarithmic amplification factor necessary for efficient parametric four-photon interaction.

The first terms in Equations 33 and 34 are responsible for the parametric four-photon interaction. the second and third terms describe the cross-phase modulation. In Equations 35 and 36 the first terms consider self-phase modulation and the second terms cause crossphase modulation. The self-phase modulations and cross-phase modulations caused by the signal and idler fields $E\left(\omega_{3^{\prime}}\right)$ and $E\left(\omega_{4^{\prime}}\right)$ are neglected.

The solution of the equation system 33-36 for no input light at $\omega_{3^{\prime}}$ and $\omega_{4^{\prime}}$ is [58] (solutions for the case of the present input signal and/or idler light are given in $[58,63]$ ):

$$
\begin{gathered}
E_{1}(l)=E_{1}(0) \exp \left(-\mathrm{i} \delta k_{1} l\right) \\
E_{R}(l)=E_{R}(0) \exp \left(-i \delta k_{R} l\right) \\
I\left(\omega_{3^{\prime}}, l\right)=I_{N}\left(\omega_{4^{\prime}}\right)\left(\left|\kappa_{3^{\prime}}\right|^{2} /\left|\beta^{\prime}\right|^{2}\right)\left|\sinh \left(\beta^{\prime} l\right)\right|^{2} \\
I\left(\omega_{4^{\prime}}, l\right)=\left.I_{N}\left(\omega_{3^{\prime}}\right)\left(\left|\kappa_{4^{\prime}}\right|^{2} /\left|\beta^{\prime}\right|^{2}\right) \sinh \left(\beta^{\prime} l\right)\right|^{2}
\end{gathered}
$$

with

$$
\begin{aligned}
\kappa_{3^{\prime}} & =\left[\omega_{3^{\prime}} / 2 n_{3^{\prime}} c_{0}\right] 6 \chi^{(3)}\left(-\omega_{3^{\prime}} ; \omega_{1}, \omega_{R},-\omega_{4^{\prime}}\right) E_{1} E_{R} \\
& =\left[\omega_{3^{\prime}} / n_{3^{\prime}}\left(n_{1} n_{R}\right)^{1 / 2} \epsilon_{0} c_{0}^{2}\right] 6 \chi^{(3)}\left(-\omega_{3^{\prime}} ; \omega_{1}, \omega_{R},-\omega_{4^{\prime}}\right)\left(I_{1} I_{R}\right)^{1 / 2} \\
\kappa_{4^{\prime}} & =\left[\omega_{4^{\prime}} / 2 n_{3^{\prime}} c_{0}\right] 6 \chi^{(3)^{*}}\left(-\omega_{3^{\prime}} ; \omega_{1}, \omega_{R},-\omega_{4^{\prime}}\right) E_{1} E_{R} \\
& =\left[\omega_{4^{\prime}} / n_{4^{\prime}}\left(n_{1} n_{R}\right)^{1 / 2} \epsilon_{0} c_{0}^{2}\right] 6 \chi^{(3)^{*}}\left(-\omega_{3^{\prime}} ; \omega_{1}, \omega_{R},-\omega_{4^{\prime}}\right)\left(I_{1} I_{R}\right)^{1 / 2}
\end{aligned}
$$




$$
\begin{gathered}
\beta^{\prime}=\left[\kappa_{3^{\prime}}^{*} \kappa_{4^{\prime}}-\frac{1}{4}\left[\Delta k-\delta k_{1}-\delta k_{R}+\delta k_{3}+\delta k_{4}\right]^{2}\right]^{1 / 2}=\left[\kappa_{3^{\prime}}^{*} \kappa_{4^{\prime}}-\frac{1}{4} \delta K^{2}\right]^{1 / 2} \\
\delta k_{1}=\frac{\omega_{1}}{2 n_{1} c_{0}} 3 \chi_{\mathrm{nl}}^{(3)}\left[\left|E_{1}\right|^{2}+2\left|E_{R}\right|^{2}\right]=\frac{\omega_{1}}{n_{1}^{2} \epsilon_{0} c_{0}^{2}} 3 \chi_{\mathrm{nl}}^{(3)}\left[I_{1}+2 \frac{n_{1}}{n_{R}} I_{R}\right] \\
\delta k_{R}=\frac{\omega_{R}}{2 n_{R} c_{0}} 3 \chi_{\mathrm{nl}}^{(3)}\left[2\left|E_{1}\right|^{2}+\left|E_{R}\right|^{2}\right]=\frac{\omega_{R}}{n_{R}^{2} \epsilon_{0} c_{0}^{2}} 3 \chi_{\mathrm{nl}}^{(3)}\left[2 \frac{n_{R}}{n_{1}} I_{1}+I_{R}\right] \\
\delta k_{3^{\prime}}=\frac{\omega_{3^{\prime}}}{2 n_{3^{\prime}} c_{0}} 6 \chi_{\mathrm{nl}}^{(3)}\left[\left|E_{1}\right|^{2}+\left|E_{R}\right|^{2}\right]=\frac{\omega_{3^{\prime}}}{n_{3^{\prime}}^{2} \epsilon_{0} c_{0}^{2}} 6 \chi_{\mathrm{nl}}^{(3)}\left[\frac{I_{1}}{n_{1}}+\frac{I_{R}}{n_{R}}\right] \\
\delta k_{4^{\prime}}=\frac{\omega_{4^{\prime}}}{2 n_{4^{\prime}} c_{0}} 6 \chi_{\mathrm{nl}}^{(3)}\left[\left|E_{1}\right|^{2}+\left|E_{R}\right|^{2}\right]=\frac{\omega_{4^{\prime}}}{n_{4^{\prime}}^{2} \epsilon_{0} c_{0}^{2}} 6 \chi_{\mathrm{nl}}^{(3)}\left[\frac{I_{1}}{n_{1}}+\frac{I_{R}}{n_{R}}\right]
\end{gathered}
$$

In the case of $\Delta k=-\left(\delta k_{3^{\prime}}+\delta k_{4^{\prime}}-\delta k_{1}-\delta k_{R}\right)$ the propagation coefficient mismatch is $\delta K=0$. The largest negative $\Delta k$ value occurs at $\left(\nu_{1}+\nu_{R}\right) / 2$. For second-harmonic pumping it is $\Delta k \approx-19 \mathrm{~cm}^{-1}$ at $\left(\tilde{\nu}_{S H}+\tilde{\nu}_{R}\right) / 2 \approx 17800 \mathrm{~cm}^{-1}$ and an intensity of $I_{S H}=I_{R} \approx 1.35 \times 10^{11} \mathrm{~W} \mathrm{~cm}^{-2}$ is necessary for propagation phase-matching $\delta K=0$ at $\tilde{\nu}_{3^{\prime}}=\tilde{\nu}_{4^{\prime}}=17800 \mathrm{~cm}^{-1}$. If the propagation coefficient mismatch is $\delta K=0$ (possible in frequency region $\tilde{\nu}_{1} \geq \tilde{\nu}_{3^{\prime}} \geq \tilde{\nu}_{4^{\prime}} \geq \tilde{\nu}_{R}$ by appropriate pump intensities $I_{1}$ and $I_{R}$ ) then the parametric amplification coefficient $\beta^{\prime}$ is maximal and given by

$$
\begin{aligned}
& \beta_{\max }^{\prime}=\left[\frac{\omega_{3^{\prime}} \omega_{4^{\prime}}}{n_{3^{\prime} n_{4^{\prime}}}}\right]^{1 / 2} \frac{3}{c_{0}} \chi^{(3)^{*}}\left(-\omega_{3^{\prime}} ; \omega_{1}, \omega_{R},-\omega_{4^{\prime}}\right)\left|E_{1}\right|\left|E_{R}\right| \\
& =\frac{6}{\epsilon_{0} c_{0}^{2}} \chi^{(3)^{*}}\left(-\omega_{3^{\prime}} ; \omega_{1}, \omega_{R},-\omega_{4^{\prime}}\right)\left[\frac{\omega_{3^{\prime}} \omega_{4^{\prime}}}{n_{1} n_{R} n_{3^{\prime}} n_{4^{\prime}}} I_{1} I_{R}\right]^{1 / 2}
\end{aligned}
$$

and Equations 39 and 40 reduce to

$$
\begin{aligned}
& I_{\max }\left(\omega_{3^{\prime}}, l\right)=I_{N}\left(\omega_{4^{\prime}}\right)\left(\omega_{3} n_{4^{\prime}} / \omega_{4} n_{3^{\prime}}\right)\left|\sinh \left(\beta_{\max }^{\prime} l\right)\right|^{2} \\
& I_{\max }\left(\omega_{4^{\prime}}, l\right)=I_{N}\left(\omega_{3^{\prime}}\right)\left(\omega_{4} n_{3^{\prime}} / \omega_{3} n_{4^{\prime}}\right)\left|\sinh \left(\beta_{\max }^{\prime} l\right)\right|^{2}
\end{aligned}
$$

The nonlinear susceptibilities for nondegenerate parametric four-photon interaction $\chi^{(3)}\left(-\omega_{3^{\prime}} ; \omega_{1}, \omega_{R},-\omega_{4^{\prime}}\right)$ and $\chi^{(3)}\left(-\omega_{4^{\prime}} ; \omega_{1}, \omega_{R},-\omega_{3^{\prime}}\right)$ are similar to the nonlinear susceptibilities for degenerate parametric four-photon interaction (Equation 11). The main difference is that resonances occur not only when $\omega_{1}-\omega_{4^{\prime}}$ approaches $\omega_{v 1}$ or $\omega_{v 2}$ but also when $\omega_{R}-\omega_{4}$, comes near to $\omega_{v 1}$ or $\omega_{v 2}$. The approximate spectral dispersion is given by

$$
\begin{aligned}
& \chi^{(3)}\left(-\omega_{3^{\prime}} ; \omega_{1}, \omega_{R},-\omega_{4^{\prime}}\right)=\chi^{(3)^{*}}\left(-\omega_{4^{\prime}} ; \omega_{1}, \omega_{R},-\omega_{3^{\prime}}\right) \\
& \approx \chi_{N R}^{(3)}+\frac{\chi_{R}^{\prime \prime}}{f} \Gamma\left[\frac{\omega_{v 1}-\omega_{1}+\omega_{4^{\prime}}-\mathrm{i} \Gamma}{\left(\omega_{v 1}-\omega_{1}+\omega_{4^{\prime}}\right)^{2}+\Gamma^{2}}+\frac{\omega_{v 2}-\omega_{1}+\omega_{4^{\prime}}-\mathrm{i} \Gamma}{\left(\omega_{v 2}-\omega_{1}+\omega_{4^{\prime}}\right)^{2}+\Gamma^{2}}\right. \\
& \left.+\frac{\omega_{v 1}-\omega_{R}+\omega_{4^{\prime}}-\mathrm{i} \Gamma}{\left(\omega_{v 1}-\omega_{R}+\omega_{4^{\prime}}\right)^{2}+\Gamma^{2}}+\frac{\omega_{v 2}-\omega_{R}+\omega_{4^{\prime}}-\mathrm{i} \Gamma}{\left(\omega_{v 2}-\omega_{R}+\omega_{4^{\prime}}\right)^{2}+\Gamma^{2}}\right]
\end{aligned}
$$


In the case of efficient pump-pulse-nondegenerate parameteric four-photon interaction the amplification $I\left(\omega_{3^{\prime}}, l\right) / I_{N}\left(\omega_{4^{\prime}}\right) \approx I\left(\omega_{4^{\prime}}, l\right) / I_{N}\left(\omega_{3^{\prime}}\right)$ has to be of the order of $10^{7}$. (see above). For this high amplification the Equations 39 and 40 reduce to

$$
\frac{I\left(\omega_{3^{\prime}}, l\right)}{I_{N}\left(\omega_{4^{\prime}}\right)} \approx \frac{I\left(\omega_{4^{\prime}}, l\right)}{I_{N}\left(\omega_{3^{\prime}}\right)} \approx\left|\exp \left(\beta^{\prime} l\right)\right|^{2}=\exp \left[2 \operatorname{Re}\left(\beta^{\prime}\right) l\right]
$$

In Fig. 21 the gain factor $G^{\prime}=2 \operatorname{Re}\left(\beta^{\prime}\right) l$ is plotted versus $\tilde{\nu}$ for $\tilde{\nu}_{1}=\tilde{\nu}_{S H}=18975 \mathrm{~cm}^{-1}$ using the nonlinear susceptibility dispersion of Fig. 20. The curves in Fig. 21 apply to different pump pulse intensities $I_{S H}=I_{R}$. In the region $\tilde{\nu}_{S H} \geq \tilde{\nu} \geq \tilde{\nu}_{R}$ the structure of the gain factor changes with pump pulse intensity. Whenever propagation phasematching, $\delta K=0$, is achieved at a certain frequency, the gain factor is rather high. As soon as efficient stimulated Stokes Raman light is generated (curve $1, I_{S H} \pm I_{R}=$ $I_{1, \text { th }}^{(R)}=6 \times 10^{10} \mathrm{~W} \mathrm{~cm}^{-2}$ ), the parametric light generation at the short-wavelength side of the Stokes line and at the long-wavelength side of the pump line is already quite effective. Outside the range $\tilde{\nu}_{S H} \geq \tilde{\nu} \geq \tilde{\nu}_{R}$ the structure of the gain factor is determined by the structure of $\chi^{(3)}$ and the gain reduces by the positive phase-mismatches $\Delta k$ (Fig. 14) and $\delta K$.

Besides enhanced collinear interaction in the region $\nu_{R}<\nu_{4^{\prime}}<\nu_{3^{\prime}}<\nu_{1}$ the longitudinally phase-matched Čerenkov-like filament-limited nondegenerate parametric fourphoton interaction contributes to the light continuum generation.

Experimentally the spectral energy density in the region $\tilde{\nu}_{R}<\tilde{\nu}<\tilde{\nu}_{S H}$ was larger than in the region $\tilde{\nu}_{S H}<\tilde{\nu}<\tilde{\nu}_{A}$. In particular, the spectrum of Fig. $10 \mathrm{~b}$ is a clear indication of an enhanced phase-matched nondegenerate parametric four-photon interaction $\tilde{\nu}_{S H}+\tilde{\nu}_{R} \rightarrow \tilde{\nu}_{3^{\prime}}+\tilde{\nu}_{4^{\prime}}$ with $\tilde{\nu}_{3^{\prime}}=\tilde{\nu}_{S H}+1850 \mathrm{~cm}^{-1}$ and $\tilde{\nu}_{4^{\prime}}=\tilde{\nu}_{R}-1850 \mathrm{~cm}^{-1}$.

\subsection{Cascading antiStokes up-conversion}

After the generation of an intense Stokes Raman pulse the generated spectrum $I\left(\omega_{(i)}\right)$ may be up-converted by the process $\omega_{1}+\omega_{(i)}-\omega_{R} \rightarrow \omega_{(i+1)}$. This process may occur in cascading order. The interaction is described by the following two equations, if absorption losses as well as pump laser and Stokes light depletion are neglected:

$$
\partial E\left(\omega_{(i)}\right) / \partial z=-\mathrm{i}\left(\omega_{(i)} / 2 n_{(i)} c_{0}\right) 6 \chi^{(3)^{*}}\left(-\omega_{(i+1)} ; \omega_{1},-\omega_{R}, \omega_{(i)}\right) E_{1}^{*} E_{R} E\left(\omega_{(i+1)}\right) \exp (\mathrm{i} \Delta \mathbf{k r})
$$

$$
\partial E\left(\omega_{(i+1)}\right) / \partial z=-\mathrm{i}\left(\omega_{(i)} / 2 n_{(i)} c_{0}\right) 6 \chi^{(3)}\left(-\omega_{(i+1)} ; \omega_{1},-\omega_{R}, \omega_{(i)}\right) E_{1} E_{R}^{*} E\left(\omega_{(i)}\right) \exp (\mathrm{i} \Delta \mathbf{k r})
$$

Under longitudinally phase-matched filamentary conditions the solution of Equations 53 and 54 gives [23] for $\mathbf{k}_{(i)}\left\|\mathbf{k}_{1}\right\| \mathbf{k}_{R}\left(\varphi_{(i)}=0, \varphi_{(i+1)} \neq 0\right)$

$$
\begin{gathered}
I\left(\omega_{(i)}\right) \approx I\left(\omega_{(i)}\right)\left|\cos ^{2}\left[g_{(i)}\left(I_{L} I_{R}\right)^{1 / 2} I_{i i}\right]\right|^{I / l_{i i}} \\
I\left(\omega_{(i+1)}\right) \approx \frac{\omega_{(i+1)}}{\omega_{(i)}} I\left(\omega_{(i)}\right)\left\{1-\left|\cos ^{2}\left[g_{(i)}\left(I_{1} I_{R}\right)^{1 / 2} l_{i i}\right]\right|^{I / l_{i i}}\right\}
\end{gathered}
$$




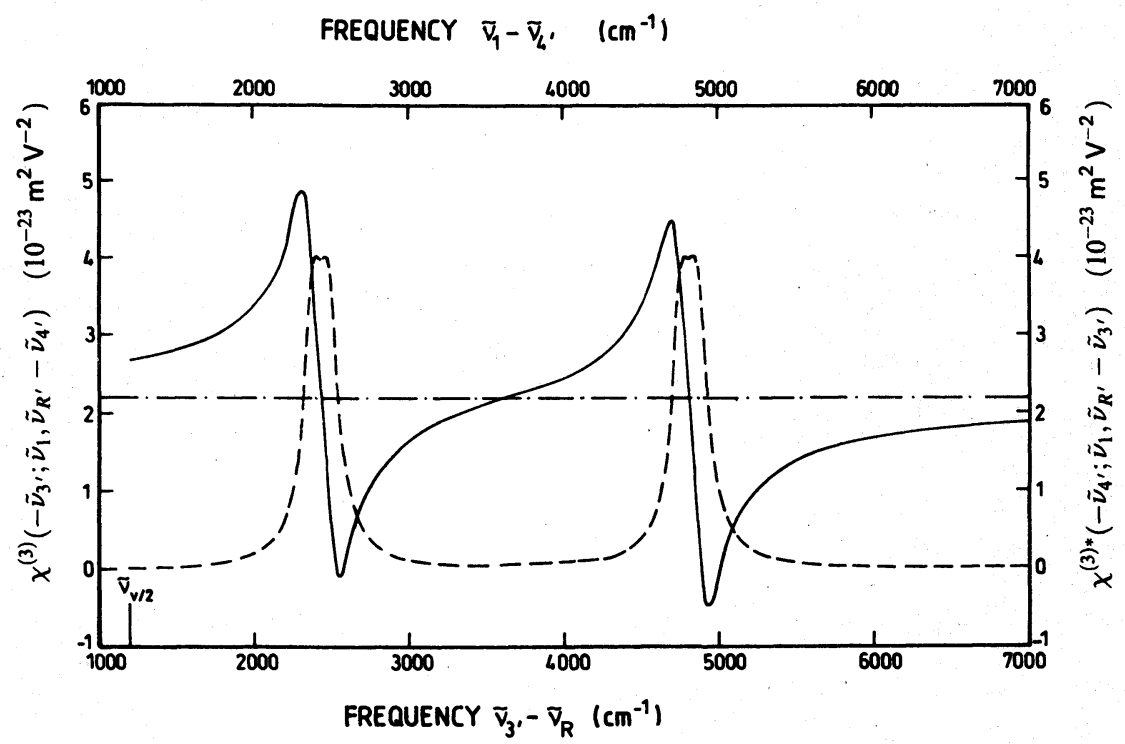

Figure 20 Frequency dependence of pump-pulse-nondegenerate third-order nonlinear susceptibilities $\chi^{(3)}\left(-\tilde{\nu}_{3^{\prime}} ; \tilde{\nu}_{1}, \tilde{\nu}_{R},-\tilde{\nu}_{4^{\prime}}\right)$ (left ordinate and low abscissa) and $\chi^{(3) *}\left(-\tilde{\nu}_{4^{\prime}} ; \tilde{\nu}_{1}, \tilde{\nu}_{R},-\tilde{\nu}_{3^{\prime}}\right)$ (right ordinate and upper abscissa, curves are identical). Solid curve, real part $\chi^{(3)^{\prime}}$. Dashed curve, imaginary part $\chi^{(3)^{\prime \prime}}$. Dash-dotted line, nonresonant contribution $\chi_{N R}^{(3)}$.

where

$$
\begin{aligned}
g_{(i)}= & \frac{12 \mathrm{i}}{\epsilon_{0} c_{0}^{2}}\left[\frac{\omega_{(i)} \omega_{(i+1)}}{n_{1} n_{R} n_{(i)} n_{(i+1)}}\right]^{1 / 2} \chi^{(3)}\left(-\omega_{(i+1)} ; \omega_{1},-\omega_{R}, \omega_{(i)}\right) \\
& \approx \frac{12}{\epsilon_{0} c_{0}^{2}}\left[\frac{\omega_{(i)} \omega_{(i+1)}}{n_{1} n_{R} n_{(i)} n_{(i+1)}}\right]^{1 / 2} \chi_{R}^{\prime \prime}
\end{aligned}
$$

$\chi^{(3)}\left(-\omega_{(i+1)} ; \omega_{1},-\omega_{R}, \omega_{(i)}\right)$ is difference-frequency enhanced since $\omega_{1}-\omega_{R}=\omega_{v}$, i.e. $\chi^{(3)}\left(-\omega_{(i+1)} ; \omega_{1},-\omega_{R}, \omega_{(i)}\right) \approx-\mathrm{i} \chi_{R}^{\prime \prime} . l_{\ddot{u}}$ is the overlap length of the generated light at $\omega_{(i+1)}$.

Efficient cascading up-conversion occurs if $G_{u c}=g_{(i)}^{2} I_{1} I_{R} l_{\dot{u}} l$ is of the order of 1 . For $I_{1}=6 \times 10^{10} \mathrm{~W} \mathrm{~cm}^{-2}, I_{R}=6 \times 10^{9} \mathrm{~W} \mathrm{~cm}^{-2}, l=5 \mathrm{~cm}, l_{\dot{u}} \approx 100 d \approx 0.5 \mathrm{~mm}$, we estimate $G \approx 1.7\left(\lambda_{1}=\lambda_{S H}=527 \mathrm{~nm} ; \tilde{\nu}_{(i)}=20700 \mathrm{~cm}^{-1}, \tilde{\nu}_{(i+1)}=23080 \mathrm{~cm}^{-1}\right)$, indicating very strong cascading Raman-induced cascading up-conversion. The repeated CARS-like peaks at the antiStokes side of the spectra shown in the Figs 3, 4, and 9 are a clear indication of the cascading antiStokes up-conversion described. The spectrum of Fig. 5 is explained by the processes $\tilde{\nu}_{L}+\tilde{\nu}_{(i)}-\tilde{\nu}_{P} \rightarrow \tilde{\nu}_{(i+1)}$ with $\tilde{\nu}_{(1)}=\tilde{\nu}_{L}+2300 \mathrm{~cm}^{-1}, \tilde{\nu}_{P}=$ $\tilde{\nu}_{L}-\delta \tilde{\nu}_{A}=\tilde{\nu}_{l}-2070 \mathrm{~cm}^{-1}$, and $i=1,2,3$, where $\tilde{\nu}_{P}$ is generated by the process $\tilde{\nu}_{L}+\tilde{\nu}_{R} \rightarrow \tilde{\nu}_{3^{\prime}}+\tilde{\nu}_{P}$ described in Section 4.6.

\section{Conclusions}

The generation of superbroadened spectra (supercontinuum generation [48]) in $\mathrm{D}_{2} \mathrm{O}$ by application of fundamental and second-harmonic pulses of a mode-locked $\mathrm{Nd}$ : glass laser has been studied under self-focusing conditions. Stimulated Raman scattering, 


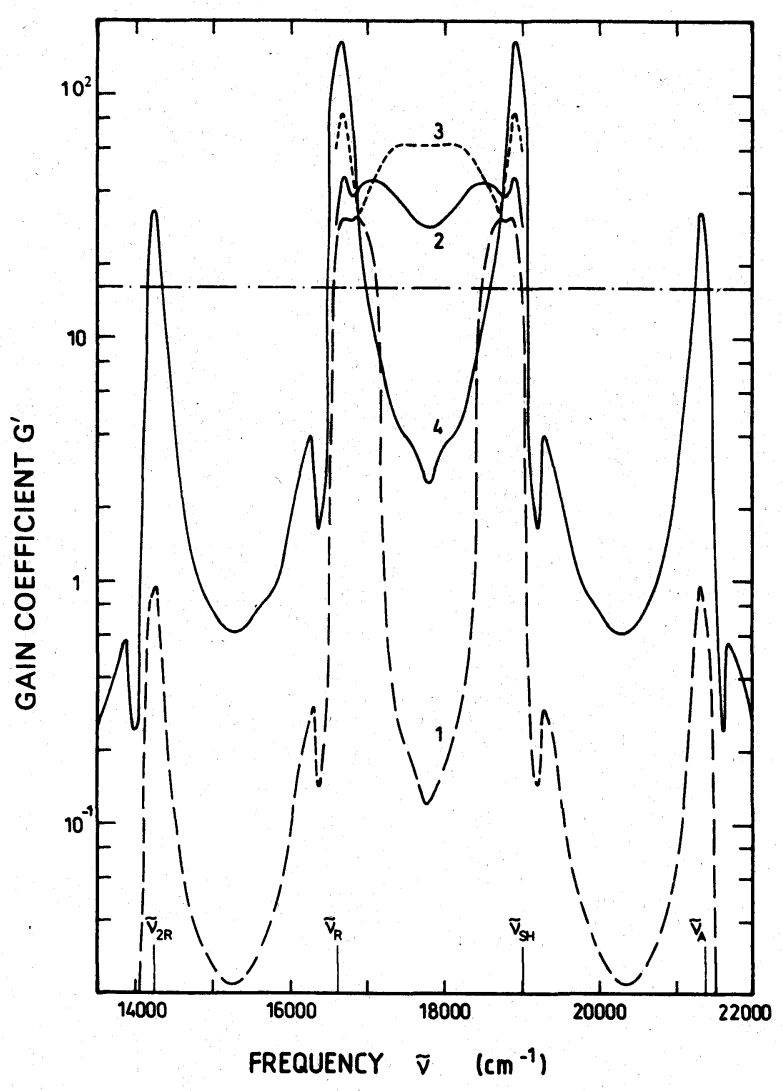

Figure 21 Gain coefficient $G^{\prime}=2 \operatorname{Re}\left(\beta^{\prime}\right) l$ of collinear parametric interaction process $\tilde{\nu}_{S H}+\tilde{\nu}_{R} \rightarrow \tilde{\nu}_{3^{\prime}}+\tilde{\nu}_{4^{\prime}}$ versus frequency $\tilde{\nu}$ $\left(\tilde{\nu}\right.$ represents $\tilde{\nu}_{3^{\prime}}$ for $\tilde{\nu} \geq\left(\tilde{\nu}_{S H}+\tilde{\nu}_{R}\right) / 2$, and $\tilde{\nu}_{4}$, for $\left.\tilde{\nu}<\left(\tilde{\nu}_{S H}+\tilde{\nu}_{R}\right) / 2\right) . \quad \tilde{\nu}_{R}=$ $\tilde{\nu}_{S H}-2380 \mathrm{~cm}^{-1}$. Curves correspond to (1) $I_{S H}=I_{R}=6 \times 10^{10} \mathrm{~W} \mathrm{~cm}^{-2}$, (2) $10^{11}$ $W \mathrm{~cm}^{-2}$, (3) $1.5 \times 10^{11} \mathrm{Wcm}^{-2}$, (4) $4 \times$ $10^{11} \mathrm{~W} \mathrm{~cm}^{-2}$.

collinear non-phase-matched stimulated parametric four-photon interaction, and longitudinally phase-matched, transversally non-phase-matched stimulated parametric fourphoton interaction occurred simultaneously in filaments formed by self-focusing. The interaction of Raman scattering and parametric four-photon interaction (also called stimulated four-photon mixing [34, 48, 51], stimulated four-wave parametric emission [50], or parametric four-photon generation [91]) has been discussed in detail. The theoretical interpretation gives a good understanding of the spectral structures of the light continua generated. The light continuum generation processes described seem to be applicable generally to picosecond light continuum generation schemes. Light continuum generation in rare gases is not influenced by Raman scattering. For femtosecond light continuum generation, stimulated Raman scattering generally plays no role because the Raman interaction becomes transient and its efficiency decreases. However, under self-focusing conditions the longitudinally phase-matched, transversally non-phase-matched Čerenkov-like stimulated parametric four-photon interaction processes also seem to be present there. In the case of picosecond light continuum generation in $\mathrm{H}_{2} \mathrm{O}$ applying fundamental pulses of a mode-locked Nd : glass laser [23], Raman scattering contributions are reduced because of a strong infrared absorption of the first Stokes Raman light $\left(\alpha\left(\tilde{\nu}_{R}\right)=\alpha\left(\tilde{\nu}_{L}-\tilde{\nu}_{v}\right)=\alpha\left(6200 \mathrm{~cm}^{-1}\right) \approx 10 \mathrm{~cm}^{-1} \quad[92], \tilde{\nu}_{v}=2 \nu_{2 s}^{\prime}=3210 \mathrm{~cm}^{-1}\right.$ and $\nu_{s s}^{\prime}=$ $\left.3405 \mathrm{~cm}^{-1}[71]\right)$. 


\section{References}

1. V. PARKASH, M. K. DHEER and T. S. JASEJA, Phys. Lett. 29A (1969) 220.

2. M. K. DHEER, D. MADHAVAN and D. R. RAO, Chem. Phys. Lett. 32 (1975) 341.

3. O. RAHN, M. MAIER and W. KAISER, Opt. Commun. 1 (1969) 109.

4. R. R. AlFANO and S. L. SHAPIRO, Phys. Rev. Lett. 24 (1970) 584, 592.

5. R. R. ALFANO and S. L. SHAPIRO, Phys. Rev. Lett. 24 (1970) 1219.

6. N. G. BONDARENKO, I. V. EREMINA and V. I. TALANOV, JETP Lett. 12 (1970) 85.

7. M. A. BOLSHOV, G. V. VENKIN, S. A. ZHILKIN and I. I. NURMINSKY, Sov. Phys. JETP 31 (1970) 1.

8. N. N. ILICHEV, V. V. KOROBKIN, V. A. KORSHUNOV, A. A. MALYUTIN, T. G. OKROASHVILI and P. P. PASHININ, JETP Lett. 15 (1972) 133.

9. W. WERNCKE, A. LAU, M. PFEIFFER, K. LENZ, H. J. WEIGMANN and T. G. THUY, Opt. Commun. 4 (1972) 413.

10. R. R. AlFANO, L. L. HOPE and S. L. SHAPIRO, Phys. Rev. A6 (1972) 433.

11. A. PENZKOFER, A. LAUBEREAU and W. KAISER, Phys. Rev. Lett. 31 (1973) 863.

12. C. A. G. O. VARMA and P. M. RENTZEPIS, J. Chem. Phys. 58 (1973) 5237.

13. G. E. BUSCH, R. P. JONES and P. M. RENTZEPIS, Chem. Phys. Lett. 18 (1973) 178.

14. M. CLERK, R. P. JONES and P. M. RENTZEPIS, Chem. Phys. Lett. 26 (1974) 167.

15. A. PENZKOFER, Opt. Commun. 11 (1974) $265^{\circ}$

16. R. R. ALFANO, J. I. GERSTEN, G. A. ZAWADZKAS and N. TZOAR, Phys. Rev. A10 (1974) 698.

17. R. H. STOLEN, IEEE J. Quantum Electron. QE-11 (1975) 100.

18. A. PENZKOFER, A. SEILMEIER and W. KAISER, Opt. Commun. 14 (1975) 363.

19. W. YU, R. R. ALFANO, C. L. SAM, and R. J. SEYMOUR, Opt. Commun. 14 (1975) 344.

20. D. K. SHARMA, R. W. YIP, D. F. WILliAMS, S. E. SUGAMORI and L. L. BRADLEY, Chem. Phys. Lett. 41 (1976) 460.

21. M. J. COLlES and G. E. WALRAFEN, Appl. Spectrosc. 30 (1976) 463.

22. W. L. SMITH, P. LIU and N. BLOEMBERGEN, Phys. Rev. A15 (1977) 2396.

23. A. PENZKOFER and W. KAISER, Opt. Quantum Electron. 9 (1977) 315.

24: D. K. SHARMA and R. W. YIP, Opt. Commun. 30 (1979) 113.

25. T. KOBAYASHI, Opt. Commun. 28 (1979) 147.

26. J. I. GERSTEN, R. R. ALFANO and M. BELIC, Phys. Rev. A21 (1980) 1222.

27. S. BELKE, R. GASE and K. VOGLER, Opt. Quantum Electron. 12 (1980) 9.

28. L. S. GOLDBERG, Picosecond Phenomena III, edited by K. B. Eisenthal, R. M. Hochstrasser, W. Kaiser and A. Laubereau, Springer Series in Chem. Phys. vol. 23 (Springer-Verlag, Berlin, 1982) p. 94.

29. A. A. MURAV'EV and A. N. RUBINOV, JETP Lett. 37 (1983) 713.

30. A. A. MURAV'EV, Sov. J. Quantum Electron. 15 (1985) 931.

31. P. B. CORKUM, P. HO, R. R. ALFANO and J. T. MANASSAH, Opt. Lett. 10 (1985) 624.

32. J. T. MANASSAH, M. A. MUSTAFA, R. R. ALFANO and P. P. HO, Phys. Lett. 113A (1985) 242.

33. P. B. CORKUM, C. ROLLAND and T. SRINIVASAN-RAO, Phys. Rev. Lett. 57 (1986) 2268.

34. R. R. ALFANO, Q. X. LI, T. JIMBO, J. T. MANASSAH and P. P. HO, Opt. Lett. 11 (1986) 626.

35. T. JIMBO, V. L. CAPLAN, Q. X. LI, Q. Z. WANG, P. P. HO and R. R. ALFANO, Opt. Lett. 12 (1987) 477.

36. P. L. BALDECK and R. R. ALFANO, J. Lightwave Technol. LT-5 (1987) 1712.

37. R. R. ALFANO, Q. Z. WANG, T. JIMBO and P. P. HO, Phys. Rev. A35 (1987) 459.

38. P. P. HO, Q. X. LI, T. JIMBO, Y. L. KU and R. R. ALFANO, Appl. Opt. 26 (1987) 2700.

39. T. M. JUDJU and L. ROTHBERG, Appl. Opt. 26 (1987) 2877.

40. R. R. ALFANO and P. P. HO, IEEE J. Quantum Electron. QE-24 (1988) 351.

41. I. GOLUB, Opt. Lett. 15 (1990) 305.

42. R. L. FORK, C. V. SHANK, C. HIRLIMANN, R. YEN and W. J. TOMLINSON, Opt. Lett. 8 (1983) 1.

43. W. H. KNOX, M. C. DOWNER, R. L. FORK and C. V. SHANK, Opt. Lett. 9 (1984) 315.

44. J. GLOWNIA. G. ARJAVALINGAM, P. SOROKIN and J. ROTHENBERG, Opt. Lett. 11 (1986) 79.

45. D. KÜHLKE, U. HERSPERS and D. VON DER LINDE, Opt. Commun. 63 (1987) 275.

46. G. R. OLBRIGHT and G. R. HARDLEY, J. Opt. Soc. Am. B6 (1989) 1363.

47. P. B. CORKUM and C. ROLLAND, IEEE J. Quantum Electron. 25 (1989) 2634.

48. R. R. ALFANO, Editor, The Supercontinuum Laser Source (Springer-Verlag, New York, 1989).

49. D. H. AUSTON, Ultrashort Light-Pulses Picosecond Techniques and Applications, edited by S. L. Shapiro, Top. in Appl. Physics, Vol. 18 (Springer-Verlag, Berlin, 1977) p. 123. 
50. A. LAUBereaU, Ultrashort Laser Pulses and Applications, edited by W. Kaiser, Top. in Appl. Phys., Vol. 60 (Springer-Verlag, Berlin, 1988) p. 35.

51. G. P. AGRAWAL, Nonlinear Fiber Optics (Academic Press, Boston, 1989).

52. S. A. AKHMANOV, V. A. VYSLOUKH and A. S. CHIRKIN, Optics of Femtosecond Laser Pulses (American Institute of Physics, New York, 1992) p. 108.

53. W. KAISER and M. MAIER, Laser Handbook, Vol. 2, Part E2, edited by F. T. Arrecchi and E. O. SchultzDubois (North-Holland, Amsterdam, 1972) p. 1077.

54. A. PENZKOFER, A. LAUBEREAU and W. KAISER, Prog. Quantum Electron. 6 (1979) 55.

55. N. Bloembergen, Opt. Commun. 8 (1973) 285.

56. N. K. DUTTA, J. Appl. Phys. 51 (1980) 84.

57. R. W. BOYD, M. G. RAYMER, P. NARUM and D. J. HARTER, Phys. Rev. A24 (1981) 411.

58. R. H. STOLEN and J. E. BJORKHOLM, IEEE J. Quantum Electron. QE-18 (1982) 1062.

59. G. YANG and Y. R. SHEN, Opt. Lett. 9 (1984) 510.

60. J. T. MANASSAH, M. A. MUSTAFA, R. R. ALFANO and P. P. HO, IEEE J. Quantum Electron. QE-22 (1986) 197.

61. D. MESTDAGH and M. HAELTERMAN, Opt. Commun. 61 (1987) 291.

62. J. HERRMANN and J. MONDRY, J. Mod. Opt. 35 (1988) 1919.

63. Y. CHEN, J. Opt. Soc. Am. B7 (1990) 43.

64. Y. R. SHEN, The Principles of Nonlinear Optics (Wiley, New York, 1984).

65. W. SCHEIDLER and A. PENZKOFER, Opt. Commun. 80 (1990) 127.

66. V. G. DIMITRIEV, G. G. GURZADYAN and D. N. NIKOGOSYAN, Handbook of Nonlinear Optical Crystals, Springer Series in Optical Sciences, Vol. 64 (Springer-Verlag, Berlin, 1991) p. 65.

67. W. BÄUMLER and A. PENZKOFER, Chem. Phys. 140 (1990) 75.

68. V. ZHELYASKOV, G. GEORGIEV, Zh. NICKOLOV and M. MITEVA, J. Raman Spectrosc. 20 (1989) 67.

69. J. W. NIBLER and G. V. KNIGHTEN, Raman Spectroscopy of Gases and Liquids, edited by A. Weber (SpringerVerlag, Berlin, 1979) p. 253.

70. A. PENZKOFER, A. BEIDOUN and S. SPEISER, Chem. Phys. 170 (1993) 139.

71. A. SOKOLOWSKA and Z. KECKI, J. Raman Spectrosc. 17 (1986) 67.

72. A. PENZKOFER and W. FALKENSTEIN, Opt. Commun. 17 (1976) 1.

73. A. C. TAM and C. K. N. PATEL, Appl. Opt. 18 (1979) 3348.

74. S. A. Sullivan, J. Opt. Soc. Am. 53 (1963) 962.

75. P. P. SETHNA, K. F. PALMER and D. WILliAMS, J. Opt. Soc. Am. 68 (1978) 815.

76. M. BAYEN, J. Phys. Rad. 3 (1942) 57.

77. P. WEIDNER and A. PENZKOFER, Opt. Quantum Electron. 25 (1993) 1.

78. G. A. ASKAR'YAN, Sov. Phys. JETP 15 (1962) 943.

79. C. H. SKINNER and P. D. KLEIBER, Phys. Rev. A21 (1980) 151.

80. E. A. CHAUCHARD and Y. H. MEYER, Opt. Commun. 52 (1984) 141.

81. I. GOlUb, G. EREZ and R. SHUKER, J. Phys. B: At. Mol. Phys. 19 (1986) L115.

82. I. GOLUB, R. SHUKER and G. EREZ, Opt. Commun. 57 (1986) 143.

83. G. I. STEGEMAN, Contemporary Nonlinear Optics, edited by G. P. Agrawal and R. W. Boyd (Academic Press, Boston, 1992) p. 1.

84. P. K. TIEN, R. UlRICH and R. J. MARTIN, Appl. Phys. Lett. 17 (1970) 447.

85. K. CHIKUMA and S. UMEGAKI, J. Opt. Soc. Am. B7 (1990) 768.

86. A. PENZKOFER and W. LEUPACHER, Opt. Quantum Electron. 20 (1988) 369.

87. M. MOSKOVitS and K. H. MiCHAEliAN, J. Chem. Phys. 69 (1978) 2306.

88. G. G. BRET, C. R. Acad. Sci. (Paris) 265 (1967) 397.

89. M. THALHAMMER and A. PENZKOFER, Appl. Phys. B32 (1983) 137.

90. I. ITZKAN and D. A. LEONHARD, Appl. Phys. Lett. 26 (1975) 106.

91. P. N. BUTCHER and D. COTTER, The Elements of Nonlinear Optics (Cambridge University Press, Cambridge, 1991).

92. F. K. PI.YI.ER and N. ACQIISTA, J. Opt. Soc. Am. 44 (1954) 505.

\section{Appendix: derivation of Equations 27 and 28}

The situation $l_{i, 4}<l_{u, 3}<l_{P} \leq l$ is considered. Within the overlap length $l_{i u, 4}$, the equations for the electric field amplitudes of the signal and idler light read [23] 


$$
\begin{aligned}
& E\left(\omega_{3}, z\right)=E\left(\omega_{3}, 0\right) \cosh (\kappa z)-\mathrm{i} E^{*}\left(\omega_{4}, 0\right)\left[n_{4} \omega_{3} / n_{3} \omega_{4}\right]^{1 / 2} \sinh (\kappa z) \\
& E\left(\omega_{4}, z\right)=E\left(\omega_{4}, 0\right) \cosh (\kappa z)-\mathrm{i} E^{*}\left(\omega_{3}, 0\right)\left[n_{3} \omega_{4} / n_{4} \omega_{3}\right]^{1 / 2} \sinh (\kappa z)
\end{aligned}
$$

Without signal and idler input the field strengths at $z=l_{i, 4}$ are

$$
\begin{aligned}
& E\left(\omega_{3}, l_{\ddot{u}, 4}\right)=-\mathrm{i} E_{N}^{*}\left(\omega_{4}\right)\left[n_{4} \omega_{3} / n_{3} \omega_{4}\right]^{1 / 2} \sinh \left(\kappa l_{i, 4}\right) \\
& E\left(\omega_{4}, l_{i, 4}\right)=-\mathrm{i} E_{N}^{*}\left(\omega_{3}\right)\left[n_{3} \omega_{4} / n_{4} \omega_{3}\right]^{1 / 2} \sinh \left(\kappa l_{i, 4}\right)
\end{aligned}
$$

and the signal and idler intensities are

$$
\begin{aligned}
& I\left(\omega_{3}, l_{\ddot{u}, 4}\right)=I_{N}\left(\omega_{4}\right)\left(\omega_{3} / \omega_{4}\right)\left|\sinh \left(\kappa l_{\ddot{u}, 4}\right)\right|^{2} \\
& I\left(\omega_{4}, l_{\ddot{u}, 4}\right)=I_{N}\left(\omega_{3}\right)\left(\omega_{4} / \omega_{3}\right)\left|\sinh \left(\kappa l_{\ddot{u}, 4}\right)\right|^{2}
\end{aligned}
$$

In the path from $z=l_{i, 4}$ to $z=2 l_{i, 4}<l_{i, 3}$ a signal input light $E\left(\omega_{3}, l_{i, 4}\right)$ is present. The signal field at $2 l_{u, 4}$ is given approximately by

$$
\begin{aligned}
E\left(\omega_{3}, 2 l_{\ddot{u}, 4}\right) & =E\left(\omega_{3}, l_{\ddot{u}, 4}\right) \cosh \left(\kappa l_{i, 4}\right)-\mathrm{i} E_{N}^{*}\left(\omega_{4}\right)\left[n_{4} \omega_{3} / n_{3} \omega_{4}\right]^{1 / 2} \sinh \left(\kappa l_{\ddot{u}, 4}\right) \\
& =-\mathrm{i} E_{N}^{*}\left(\omega_{4}\right)\left[n_{4} \omega_{3} / n_{3} \omega_{4}\right]^{1 / 2} \sinh \left(\kappa l_{\ddot{u}, 4}\right)\left[1+\cosh \left(\kappa l_{\ddot{u}, 4}\right)\right]
\end{aligned}
$$

The second part of Equation A7 was obtained by insertion of Equation A3. At $z=l_{i, 3}$ the signal field strength has grown to

$$
\begin{aligned}
E\left(\omega_{3}, l_{\ddot{u}, 3}\right)= & -\mathrm{i} E_{N}^{*}\left(\omega_{4}\right)\left[n_{4} \omega_{3} / n_{3} \omega_{4}\right]^{1 / 2} \sinh \left(\kappa l_{\ddot{u}, 4}\right) \\
& \times\left\{1+\cosh \left(\kappa l_{\ddot{u}, 4}\right)+\ldots+\left[\cosh \left(\kappa l_{\ddot{u}, 4}\right)\right]^{l_{u, 3} / l_{u, 4}-1}\right\} \\
= & -\mathrm{i} E_{N}^{*}\left(\omega_{4}\right)\left[n_{4} \omega_{3} / n_{3} \omega_{4}\right]^{1 / 2} \sinh \left(\kappa l_{i, 4}\right) \frac{\left[\cosh \left(\kappa l_{\ddot{u}, 4}\right)\right]^{l_{u}, 3} / l_{u, 4}-1}{\cosh \left(\kappa l_{\dot{u}, 4}\right)-1}
\end{aligned}
$$

The second part of the equation gives the solution of the geometric row present in the first part of the equation. The signal intensity is

$$
I\left(\omega_{3}, l_{i, 3}\right)=I_{N}\left(\omega_{4}\right)\left(\omega_{3} / \omega_{4}\right)\left|\sinh \left(\kappa l_{\ddot{u}, 4}\right)\right|^{2}\left|\frac{\left[\cosh \left(\kappa l_{i, 4}\right)\right]^{l_{i, 3} / l_{i, 4}}-1}{\cosh \left(\kappa l_{i, 4}\right)-1}\right|^{2}
$$

Along the way from $l_{i, 3}$ to $l$ the light generated in intervals of $l_{i, 3}$ is out of the interaction region. It is no longer modified and contributes linearly to the final signal. At $z=l$ the signal has grown to

$$
I\left(\omega_{3}, l\right)=I\left(\omega_{3}, l_{i, 3}\right) \frac{l_{P}}{l_{i u, 3}}
$$

where $l_{P} \leq l$ is the effective length of high pump light intensity (filament length). 
The idler signal is repeatedly generated over intervals of $l_{i, 4}$ and at the end of the sample all spectral intensity components add up. At $z=l_{\ddot{u}, 3}$ it is

$$
\begin{aligned}
& I\left(\omega_{4}, l_{\dot{u}, 3}\right)=\frac{\omega_{4}}{\omega_{3}}\left|\sinh \left(\kappa l_{\ddot{u}, 4}\right)\right|^{2}\left\{I_{N}\left(\omega_{3}\right)+I\left(\omega_{3}, l_{u, 4}\right)+I\left(\omega_{3}, 2 l_{\ddot{u}, 4}\right)+\ldots+I\left(\omega_{3},\left[\frac{l_{\ddot{u}, 3}}{l_{\ddot{u}, 4}}-1\right] l_{i, 4}\right)\right\} \\
& =\frac{\omega_{4}}{\omega_{3}}\left|\sinh \left(\kappa l_{i, 4}\right)\right|^{2}\left\{I_{N}\left(\omega_{3}\right)+I_{N}\left(\omega_{4}\right) \frac{\omega_{3}}{\omega_{4}}\left|\sinh \left(\kappa l_{i, 4}\right)\right|^{2}\left\{1+\left|1+\cosh \left(\kappa l_{i, 4}\right)\right|^{2}\right.\right. \\
& +\left|1+\cosh \left(\kappa l_{i, 4}\right)+\cosh ^{2}\left(\kappa l_{i, 4}\right)\right|^{2}+\ldots+\mid 1+\cosh \left(\kappa l_{i, 4}\right) \\
& \left.\left.+\ldots+\left.\cosh ^{l_{i, 3} / l_{u, 4}-2}\left(\kappa l_{i, 4}\right)\right|^{2}\right\}\right\} \\
& =\left|\sinh \left(\kappa l_{i, 4}\right)\right|^{2}\left\{\frac{\omega_{4}}{\omega_{3}} I_{N}\left(\omega_{3}\right)+I_{N}\left(\omega_{4}\right)\left|\sinh \left(\kappa l_{\ddot{u}, 4}\right)\right|^{2}\left[1+\left|\cosh \left(\kappa l_{i, 4}\right)-1\right|^{-2}\right.\right. \\
& \left.\times\left[\left|\cosh ^{2}\left(\kappa l_{i, 4}\right)-1\right|^{2}+\left|\cosh ^{3}\left(\kappa l_{i, 4}\right)-1\right|^{2}+\ldots+\left|\cos ^{l_{i, 3} / l_{i, 4}-1}\left(\kappa l_{u, 4}\right)-1\right|^{2}\right]\right\} \\
& =\left|\sinh \left(\kappa l_{\dot{u}, 4}\right)\right|^{2}\left\{\frac{\dot{\omega}_{4}}{\omega_{3}} I_{N}\left(\omega_{3}\right)+I_{N}\left(\omega_{4}\right)\left|\sinh \left(\kappa l_{u, 4}\right)\right|^{2}\left[1+\left|\cosh \left(\kappa l_{i, 4}\right)-1\right|^{-2}\right.\right. \\
& \times\left[\left|\cosh \left(\kappa l_{i, 4}\right)\right|^{4}-\cosh ^{2}\left(\kappa l_{i, 4}\right)-\cosh ^{* 2}\left(\kappa l_{i, 4}\right)+1\right. \\
& +\left|\cosh \left(\kappa l_{i, 4}\right)\right|^{6}-\cosh ^{3}\left(\kappa l_{\ddot{u}, 4}\right)-\cosh ^{* 3}\left(\kappa l_{i, 4}\right)+1+\ldots \\
& \left.\left.\left.+\left|\cosh \left(\kappa l_{i, 4}\right)\right|^{2\left(l_{i, 3} / l_{i, 4}-1\right)}-\cosh ^{l_{i, 3} / l_{i, 4}-1}\left(\kappa l_{i, 4}\right)-\cosh ^{* l_{i, 3} / l_{i, 4}-1}\left(\kappa l_{i, 4}\right)+1\right]\right]\right\} \\
& =\left|\sinh \left(\kappa l_{\ddot{u}}\right)\right|^{2}\left\{I_{N}\left(\omega_{3}\right) \frac{\omega_{4}}{\omega_{3}}+I_{N}\left(\omega_{4}\right)\left|\sinh \left(\kappa l_{\ddot{u}}\right)\right|^{2}\left[1+\left|\cosh \left(\kappa l_{\ddot{u}, 4}\right)-1\right|^{-2}\right.\right. \\
& \times\left[\frac{l_{i, 3}}{l_{i, 4}}-2+\left|\cosh \left(\kappa l_{\ddot{u}, 4}\right)\right|^{2} \frac{\left|\cosh \left(\kappa l_{i, 4}\right)\right|^{2\left(l_{i, 3} / l_{u, 4}-1\right)}-1}{\left|\cosh \left(\kappa l_{\ddot{u}, 4}\right)\right|^{2}-1}\right. \\
& -\cosh \left(\kappa l_{\ddot{u}, 4}\right) \frac{\cosh ^{l_{i, 3} / l_{i, 4}-1}\left(\kappa l_{\ddot{u}, 4}\right)-1}{\cosh \left(\kappa l_{i, 4}\right)-1} \\
& \left.\left.-\cosh ^{*}\left(\left(\kappa l_{i, 4}\right) \frac{\cosh ^{* l_{i, 3} / l_{i, 4}-1}\left(\kappa l_{i, 4}\right)-1}{\cosh ^{*}\left(\kappa l_{u, 4}\right)-1}\right)\right]\right\}
\end{aligned}
$$

Finally, at $z=l$ the idler signal is

$$
I_{4}\left(\omega_{4}, l\right)=I\left(\omega_{4}, l_{\ddot{u}, 3}\right) \frac{l_{P}}{l_{\ddot{l}, 3}}
$$

